\title{
Insights into the Mechanism and Kinetics of Thermo-Oxidative Degradation of HFPE High Performance Polymer
}

Sooraj Kunnikuruvan ${ }^{\dagger}$, Priya V. Parandekar ${ }^{\ddagger},{\text { Om } \text { Prakash }^{\ddagger} \text {, Thomas K. Tsotsis }}^{\perp}$, Nisanth N. Nair ${ }^{\dagger, *}$

† Department of Chemistry, Indian Institute of Technology Kanpur, Kanpur -208016, India

${ }^{\ddagger}$ Boeing Research \& Technology, India-centre, Bangalore-560016, India

${ }^{\perp}$ Boeing Research \& Technology, Huntington Beach, CA-92647, USA

\section{Supporting Information}

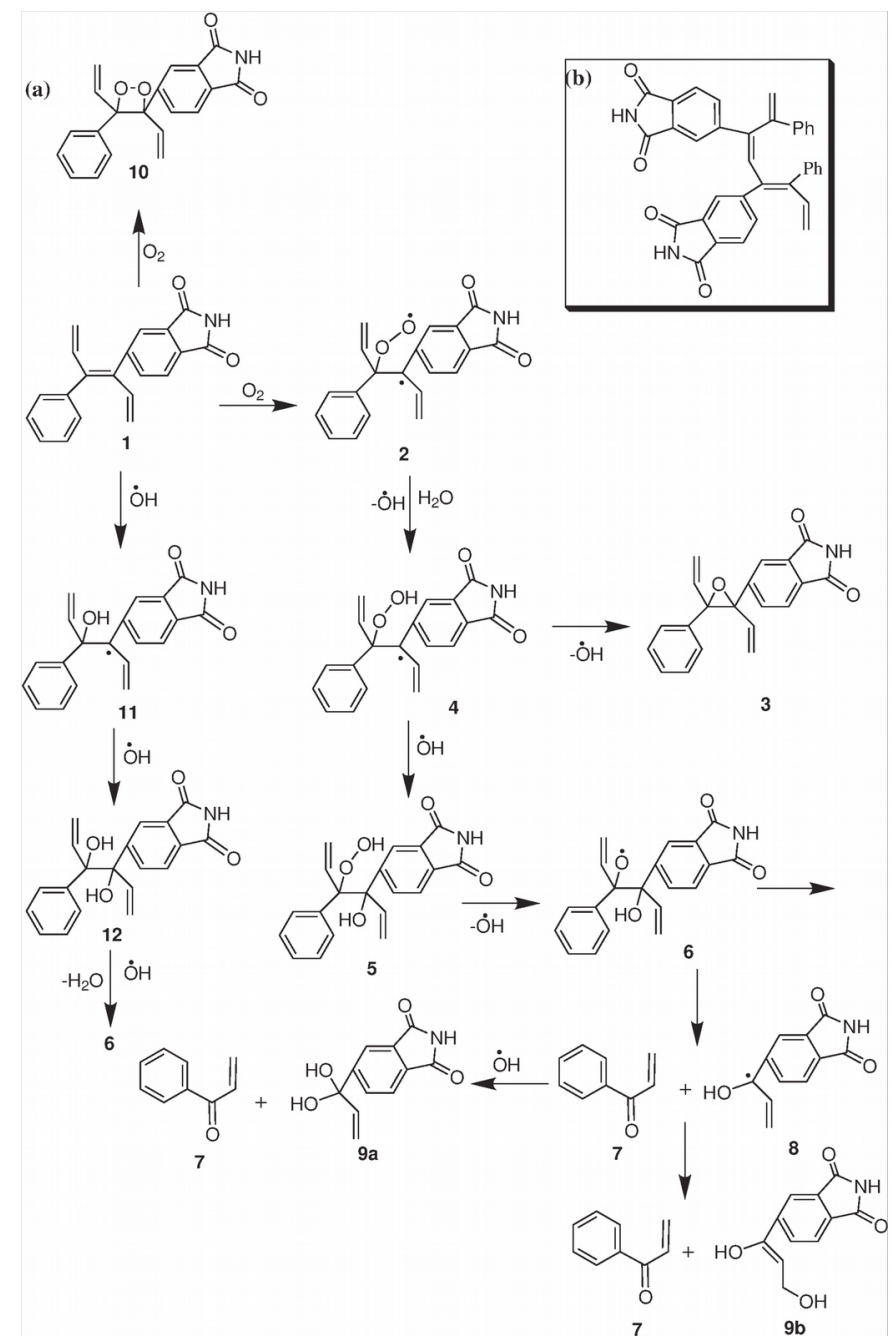

Figure S1: (a) Oxidation routes identified for PETI (1) component of HFPE (b) The chemical structure of 1 used for the quantum mechanical calculations. 


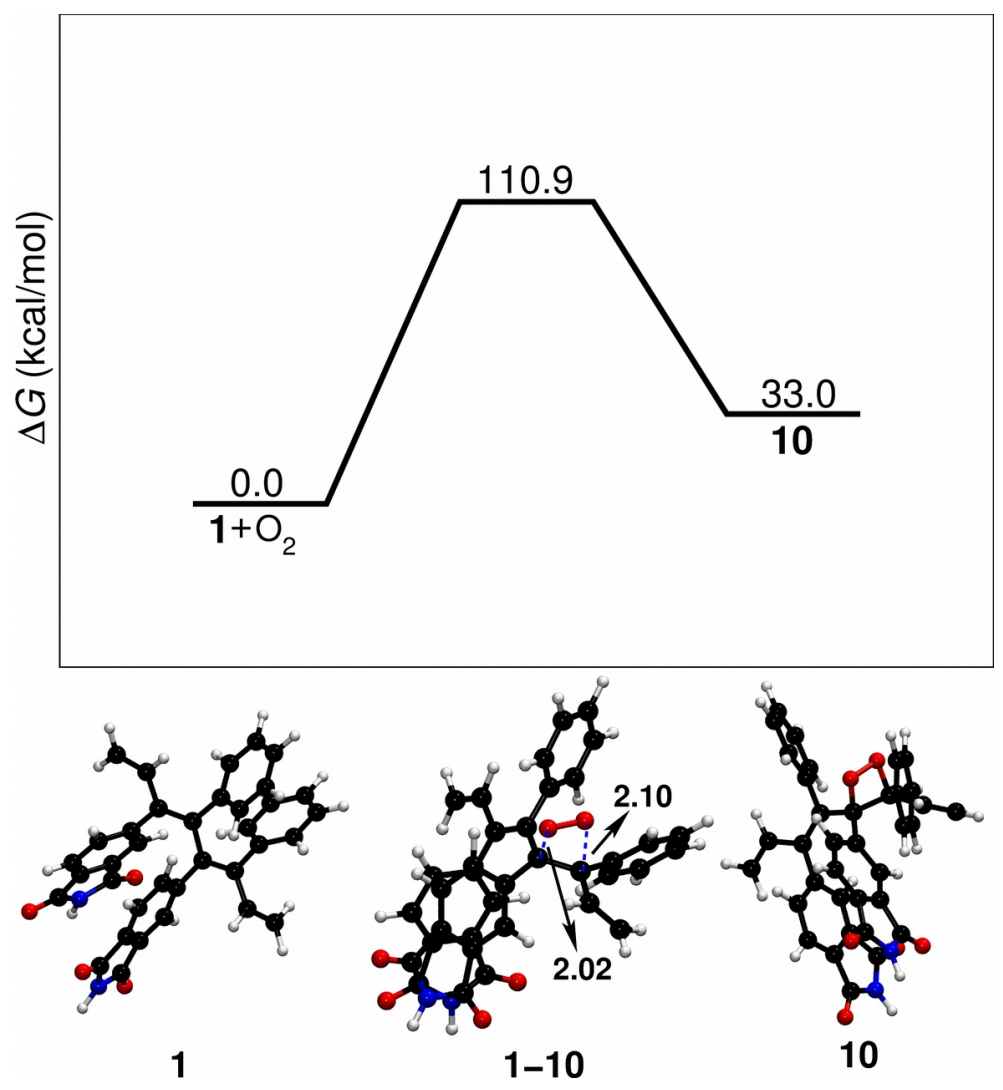

Figure S2: Free energy profile and optimized structures with important distance (in $\AA$ ) labeled for step $\mathbf{1} \rightarrow \mathbf{1 0}$ are shown. The label of the type A-B indicates that it is a transition state for the step $\mathbf{A} \rightarrow \mathbf{B}$. Atom color codes C (black), O (red), $\mathrm{N}$ (blue) and $\mathrm{H}$ (white). 

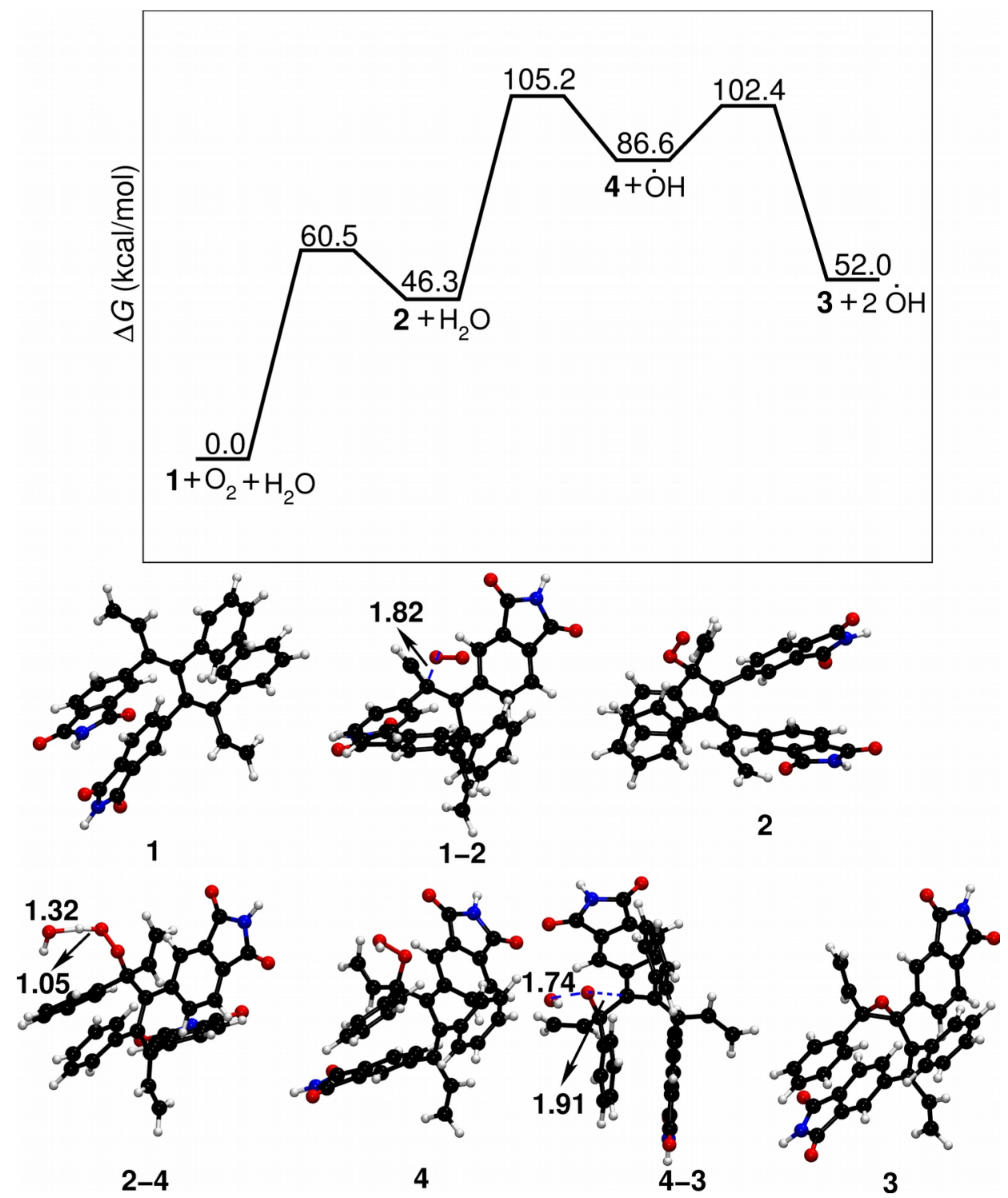

Figure S3: Free energy profile and optimized structures involve in the oxidation pathway which results in the epoxide (3) formation by PETI oxidation. 


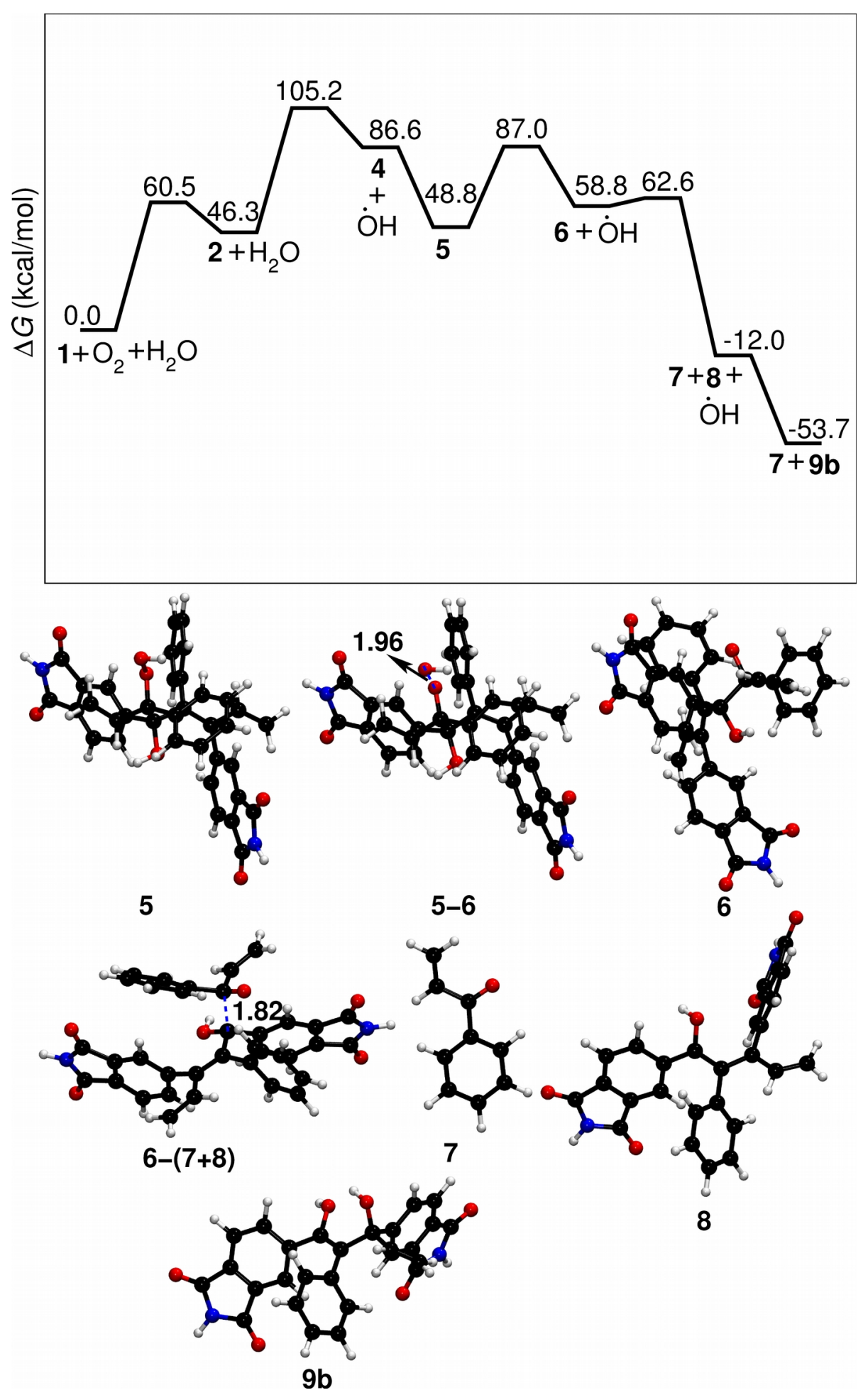

Figure S4: Free energy profile and optimized structures involve in the oxidation pathway which results in the formation of 9b by PETI oxidation. 

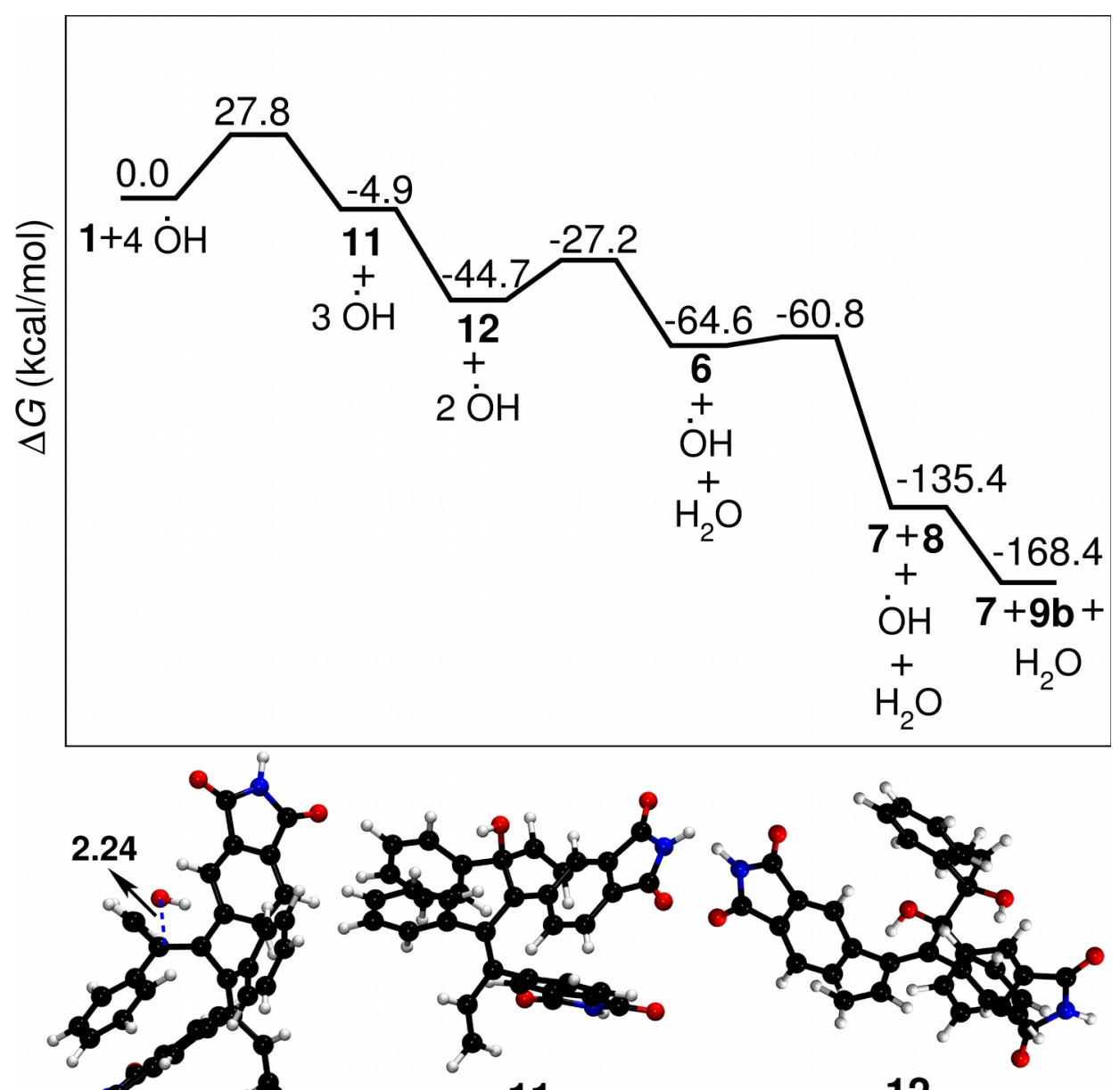

1-11

11

12

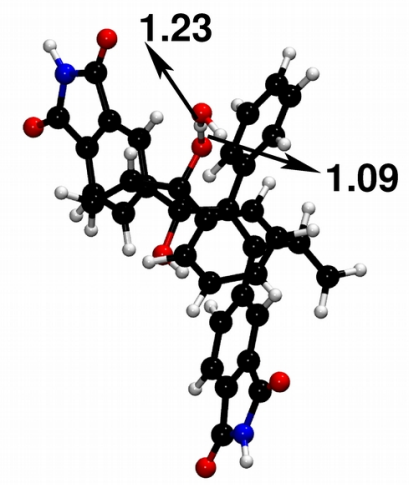

12-6

Figure S5: Free energy profile and optimized structures involve in the oxidation routes initiated by $\mathrm{OH}$ to form $\mathbf{9 b}$ and $\mathbf{7}$ from PETI. 
Table S1: Free energies (in au) computed using MPW1K/6-31+G (d,p) level of theory at $600 \mathrm{~K}$ for the PETI oxidation. Here, the label of the type A-B indicates that it is a transition state for the step $\mathbf{A} \rightarrow \mathbf{B}$.

\begin{tabular}{|c|c|}
\hline & Free energy (au) \\
\hline 1 & -1795.931830 \\
\hline 1-10 & -1946.058745 \\
\hline 10 & -1946.182894 \\
\hline $1-2$ & -1946.139048 \\
\hline 2 & -1946.161699 \\
\hline $2-4$ & -2022.490347 \\
\hline 4 & -1946.778060 \\
\hline $4-3$ & -1946.752838 \\
\hline 3 & -1871.091238 \\
\hline 5 & -2022.580314 \\
\hline $5-6$ & -2022.519459 \\
\hline 6 & -1946.838296 \\
\hline $6-7$ & -1946.832254 \\
\hline 7 & -422.807576 \\
\hline 8 & -1524.143476 \\
\hline $9 a$ & -1599.951947 \\
\hline $9 b$ & -1599.937994 \\
\hline 11 & -1946.182894 \\
\hline 1-11 & -1871.629549 \\
\hline 12 & -1871.681616 \\
\hline 12 & -1947.487073 \\
\hline $12-6$ & -2023.201149 \\
\hline $\mathrm{O}_{2}$ & -150.303690 \\
\hline ÖH & -75.741985 \\
\hline $\mathrm{H}_{2} \mathrm{O}$ & -76.422428 \\
\hline
\end{tabular}


Table S2: Free energy barriers (in kcal/mol) computed by employing MPW1K/6-31+G(d,p) level of theory at $600 \mathrm{~K}$ for PETI oxidation.

\begin{tabular}{|c|c|c|}
\hline & \multicolumn{2}{|c|}{$\begin{array}{c}\text { Free energy barrier } \\
\text { (kcal/mol) }\end{array}$} \\
\hline & Forward & Reverse \\
\hline $\mathbf{1 - 1 0}$ & 110.93 & 77.90 \\
\hline $\mathbf{1 - 2}$ & 60.54 & 14.21 \\
\hline $\mathbf{2 - 4}$ & 58.85 & 18.64 \\
\hline $\mathbf{4 - 3}$ & 15.83 & 50.44 \\
\hline $\mathbf{4 - 5}$ & 0.00 & 37.82 \\
\hline $\mathbf{5 - 6}$ & 38.19 & 38.17 \\
\hline $\mathbf{6 - ( 7 + 8 )}$ & 3.79 & 74.55 \\
\hline $\mathbf{8 - 9 a}$ & 0.00 & 32.96 \\
\hline $\mathbf{8 - 9 b}$ & 0.00 & 41.72 \\
\hline $\mathbf{1 - 1 1}$ & 27.78 & 32.67 \\
\hline $\mathbf{1 1 - 1 2}$ & 0.00 & 39.83 \\
\hline $\mathbf{1 2 - 6}$ & 17.51 & 37.38 \\
\hline
\end{tabular}


(a)<smiles>CC1C=CC=CC1</smiles><smiles>CC(C)O</smiles><smiles>c1ccccc1</smiles>

13<smiles>C1C[C@H]2CC[C@H]1C2</smiles><smiles>CC1C=CC=CC1O</smiles>

$19 \mathrm{OH}$

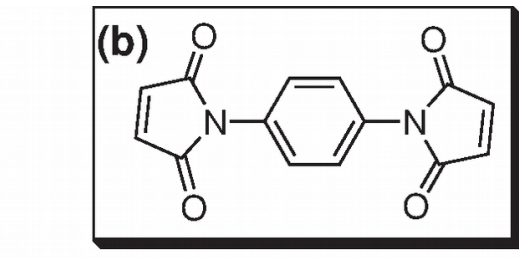<smiles>[O]OC1C=CC=CC1</smiles>

15

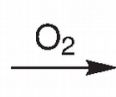

$\left\langle\begin{array}{l}0 \\ 0\end{array}\right.$

14

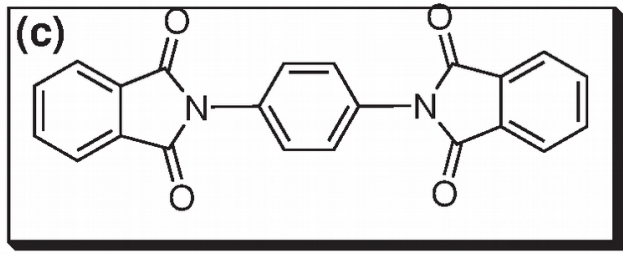

$$
\underset{-\stackrel{\mathrm{OH}}{\longrightarrow}}{\stackrel{\mathrm{H}_{2} \mathrm{O}}{\longrightarrow}}
$$<smiles>OOC1C=CC=CC1</smiles>

16

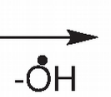

Figure S6: (a) Oxidation mechanism of BNS (13) component of HFPE (b) Chemical structure of $\mathbf{1 3}$ used in quantum mechanical calculation for all steps except step $\mathbf{1 3} \rightarrow \mathbf{1 4}$ (c) Chemical structure of $\mathbf{1 3}$ used in quantum mechanical calculation for the step $\mathbf{1 3} \rightarrow \mathbf{1 4}$. 


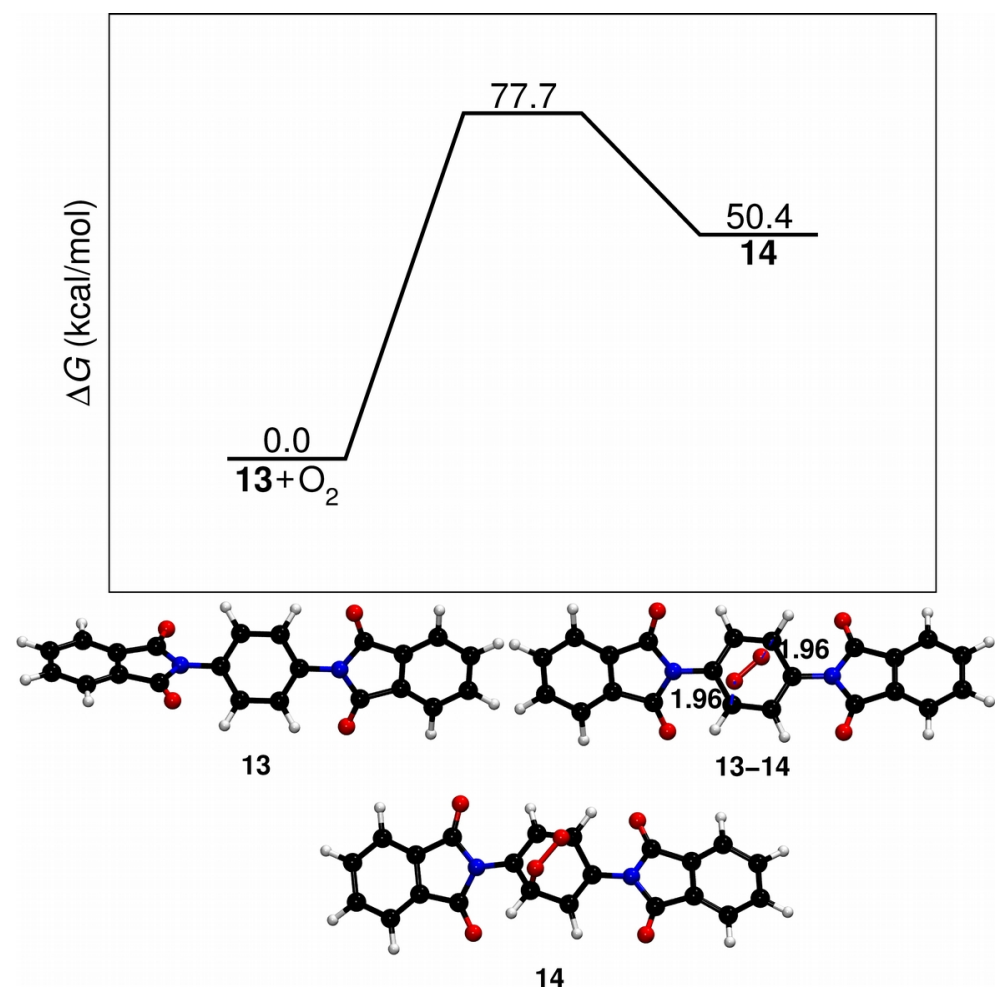

Figure S7: Free energy profile and optimized structures involve in the oxidation of BNS along step $\mathbf{1 3} \rightarrow \mathbf{1 4}$. 


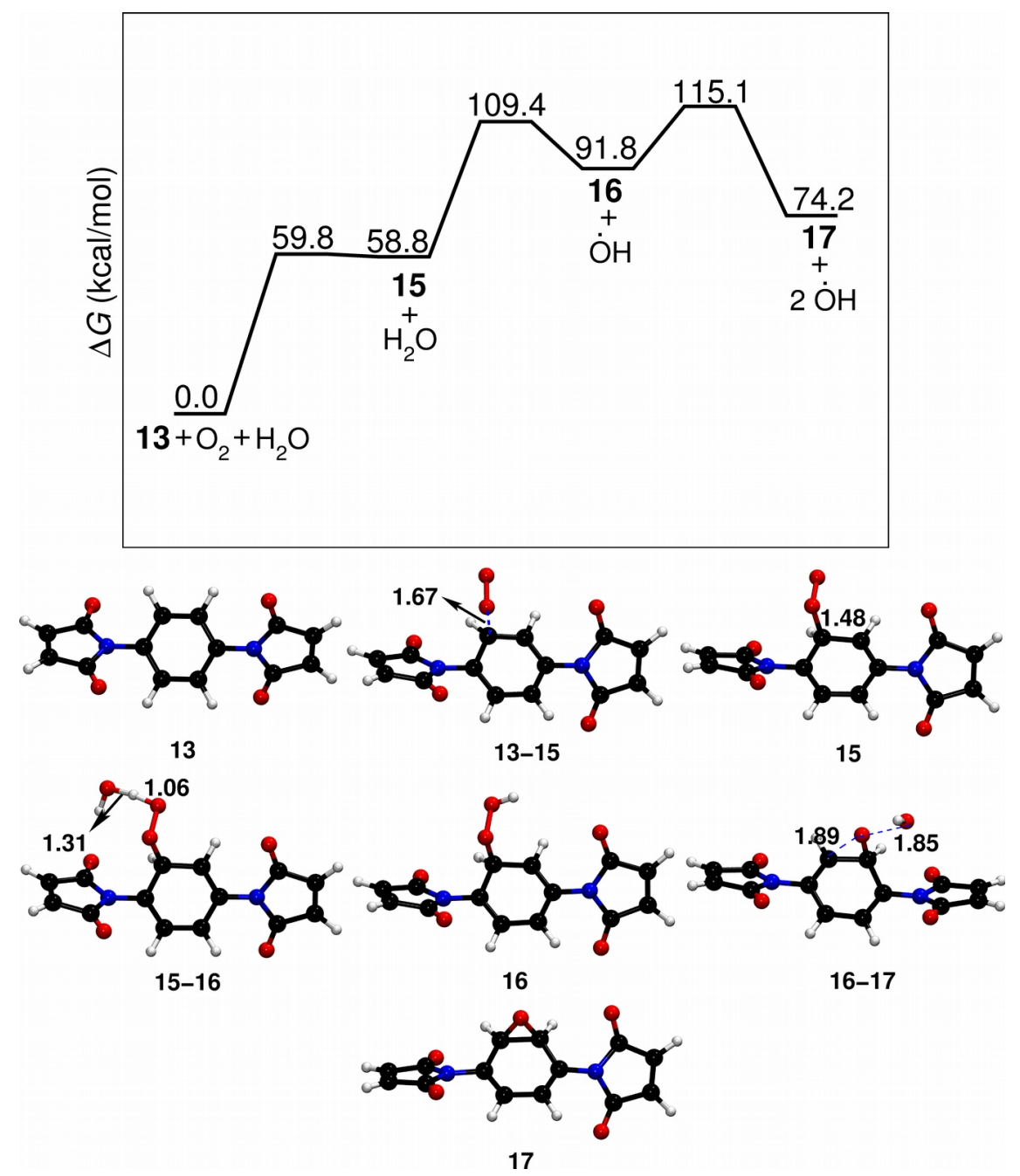

Figure S8: Free energy profile and optimized structures involve in the oxidation pathway which results in the formation of epoxide (17) from BNS. 


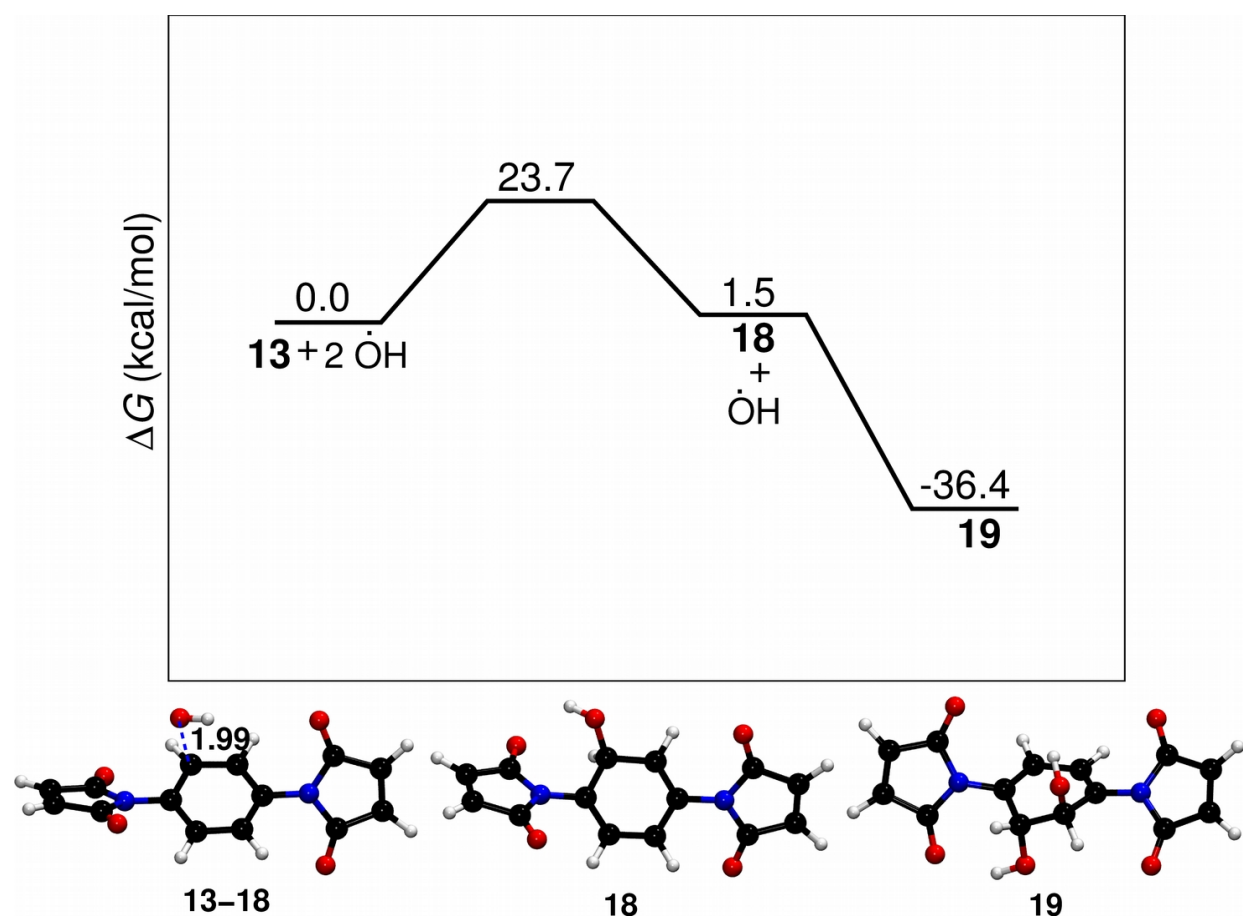

Figure S9: Free energy profile and optimized structures involve in the ÖH initiated oxidation route of BNS.

Table S3: Free energies (in au) computed using MPW1K/6-31+G (d,p) level of theory at $600 \mathrm{~K}$ for the BNS oxidation.

\begin{tabular}{|c|c|}
\hline & Free energy (au) \\
\hline $\mathbf{1 3}$ & -948.359406 \\
\hline${ }^{*} \mathbf{1 3 a}$ & -1255.546178 \\
\hline $\mathbf{1 3 a}-\mathbf{1 4}$ & -1405.725996 \\
\hline $\mathbf{1 4}$ & -1405.769532 \\
\hline $\mathbf{1 3 - 1 5}$ & -1098.567832 \\
\hline $\mathbf{1 5}$ & -1098.569411 \\
\hline $\mathbf{1 5}-\mathbf{1 6}$ & -1174.911274 \\
\hline $\mathbf{1 6}$ & -1099.197273 \\
\hline $\mathbf{1 6 - 1 7}$ & -1099.160134 \\
\hline $\mathbf{1 7}$ & -1023.483336 \\
\hline $\mathbf{1 3 - 1 8}$ & -1024.070014 \\
\hline $\mathbf{1 8}$ & -1024.096502 \\
\hline $\mathbf{1 9}$ & -1099.905870 \\
\hline $\mathrm{O}_{2}$ & -150.303690 \\
\hline ÖH & -75.741985 \\
\hline
\end{tabular}




\begin{tabular}{|c|c|}
\hline $\mathrm{H}_{2} \mathrm{O}$ & -76.422428 \\
\hline 13a was used for step $\mathbf{1 3} \rightarrow \mathbf{1 4}$. For all other calculations $\mathbf{1 3}$ was used
\end{tabular}

Table S4: Free energy barriers (in kcal/mol) computed by employing MPW1K/6-31+G(d, p) level of theory at $600 \mathrm{~K}$ for BNS.

\begin{tabular}{|c|c|c|}
\hline \multirow{2}{*}{ Step } & \multicolumn{2}{|c|}{ Free energy barrier (kcal/mol) } \\
\cline { 2 - 3 } & Forward & Reverse \\
\hline \multirow{2}{*}{$\mathbf{1 3 - 1 4}$} & 77.73 & 27.32 \\
\hline $\mathbf{1 3 - 1 5}$ & 59.78 & 0.99 \\
\hline $\mathbf{1 5 - 1 6}$ & 50.56 & 17.56 \\
\hline $\mathbf{1 6 - 1 7}$ & 23.31 & 40.91 \\
\hline $\mathbf{1 3 - 1 8}$ & 19.69 & 16.62 \\
\hline $\mathbf{1 8 - 1 9}$ & 0.00 & 42.28 \\
\hline
\end{tabular}

${ }^{*}$ 13a was used to compute the free energy barrier for step $\mathbf{1 3} \rightarrow \mathbf{1 4}$. 
(a)

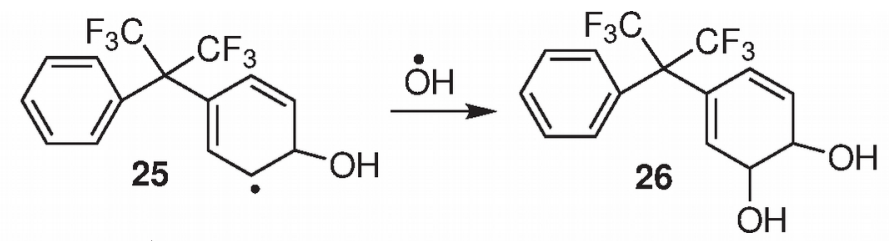

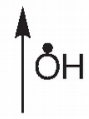<smiles>CC(c1ccccc1)(c1ccc(C(F)(F)F)cc1)C(F)(F)F</smiles><smiles>[Te][Te]</smiles>

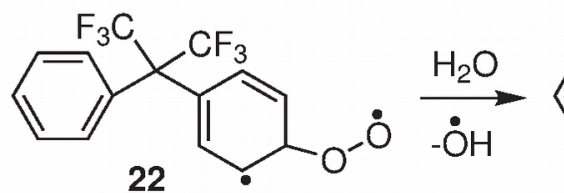<smiles>CC(C)(C1=CCC(OO)C=C1)c1ccccc1</smiles>

-ÖH

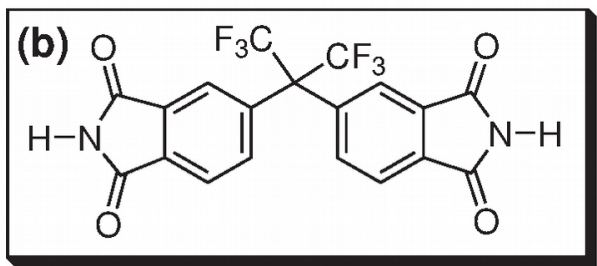<smiles>FC(F)(F)C(F)(F)c1ccccc1</smiles>

Figure S10: (a) Oxidation routes, which are initiated by $\mathrm{O}_{2}$, identified for 6FDA (20) component of HFPE. (b) Chemical structure of $\mathbf{2 0}$ used in quantum mechanical calculation. The same molecule is used to study oxidation routes involving C$\mathrm{CF}_{3}$ and $\mathrm{C}-\mathrm{F}$ bond breaking. 

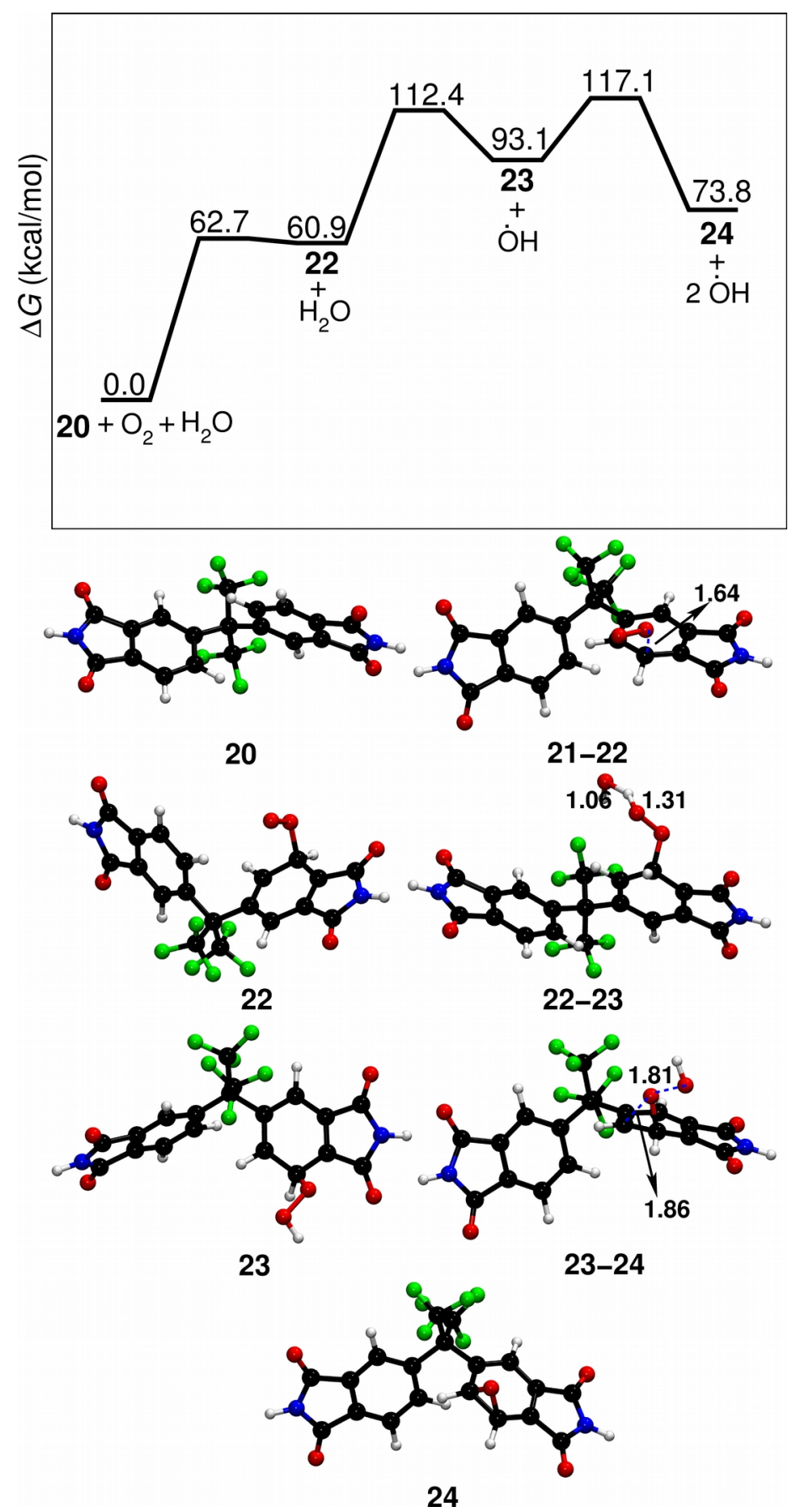

Figure S11: Free energy profile and optimized structures along the oxidation route which result in epoxide (24) formation from 6FDA. 
Table S5: Free energies (in au) for the 6FDA oxidation computed using MPW1K/6-31+G(d,p) level of theory at $600 \mathrm{~K}$. Ipso, ortho, meta and para in parenthesis indicate the position of $\mathrm{O}_{2}$ attack.

\begin{tabular}{|c|c|}
\hline & Free energy (au) \\
\hline $\mathbf{2 0}$ & -1737.794881 \\
\hline $\mathbf{2 0} \mathbf{2 1}$ & -1887.967818 \\
\hline $\mathbf{2 0 - 2 2}$ (ipso) & -1888.007540 \\
\hline $\mathbf{2 0 - 2 2}$ (ortho) & -1887.977505 \\
\hline $\mathbf{2 0 - 2 2}$ (meta) & -1887.998732 \\
\hline $\mathbf{2 0 - 2 2}$ (para) & -1887.993321 \\
\hline $\mathbf{2 2}$ (ipso) & -1887.979854 \\
\hline $\mathbf{2 2}$ (ortho) & -1887.996050 \\
\hline $\mathbf{2 2}$ (meta) & -1888.001664 \\
\hline $\mathbf{2 2}$ (para) & -1887.993406 \\
\hline $\mathbf{2 2 - 2 3}$ & -1964.342064 \\
\hline $\mathbf{2 3}$ & -1888.630894 \\
\hline${ }^{*} \mathbf{2 3 - 2 4}$ & -1888.589469 \\
\hline${ }^{*} \mathbf{2 3 - 2 4}$ & -1888.592598 \\
\hline${ }^{*} \mathbf{2 4}$ & -1812.913468 \\
\hline${ }^{\#} \mathbf{2 4}$ & -1812.919615 \\
\hline $\mathbf{2 0 - 2 5}$ & -1813.499112 \\
\hline $\mathbf{2 5}$ & -1813.534476 \\
\hline $\mathbf{2 6}$ & -1889.336814 \\
\hline${ }^{*}$ epoxide ring formation at the bridgehead \\
\hline & -18969 formation at ortho to CF3 group \\
\hline
\end{tabular}

Table S6: Free energy barriers (in kcal/mol) for 6FDA oxidation computed by employing MPW1K/6-31+G(d,p) level of theory at $600 \mathrm{~K}$.

\begin{tabular}{|c|c|c|}
\hline \multirow{2}{*}{ Step } & Free energy barrier $(\mathbf{k c a l} / \mathbf{m o l})$ \\
\cline { 2 - 3 } & Forward & Reverse \\
\hline $\mathbf{2 0 - 2 1}$ & 82.05 & 24.93 \\
\hline $\mathbf{2 0 - 2 2}$ (ipso) & 75.97 & 1.47 \\
\hline $\mathbf{2 0 - 2 2}$ (ortho) & 64.70 & 0.66 \\
\hline $\mathbf{2 0 - 2 2}$ (meta) & 62.65 & 1.84 \\
\hline $\mathbf{2 0 - 2 2}$ (para) & 66.04 & 0.05 \\
\hline $\mathbf{2 2}$ (meta)-23 & 51.47 & 19.34 \\
\hline
\end{tabular}




\begin{tabular}{|c|c|c|}
\hline $\mathbf{2 3 - 2 4}$ & 24.03 & 43.30 \\
\hline $\mathbf{2 0 - 2 5}$ & 23.69 & 22.19 \\
\hline $\mathbf{2 5 - 2 6}$ & 0.00 & 37.87 \\
\hline
\end{tabular}

(a)
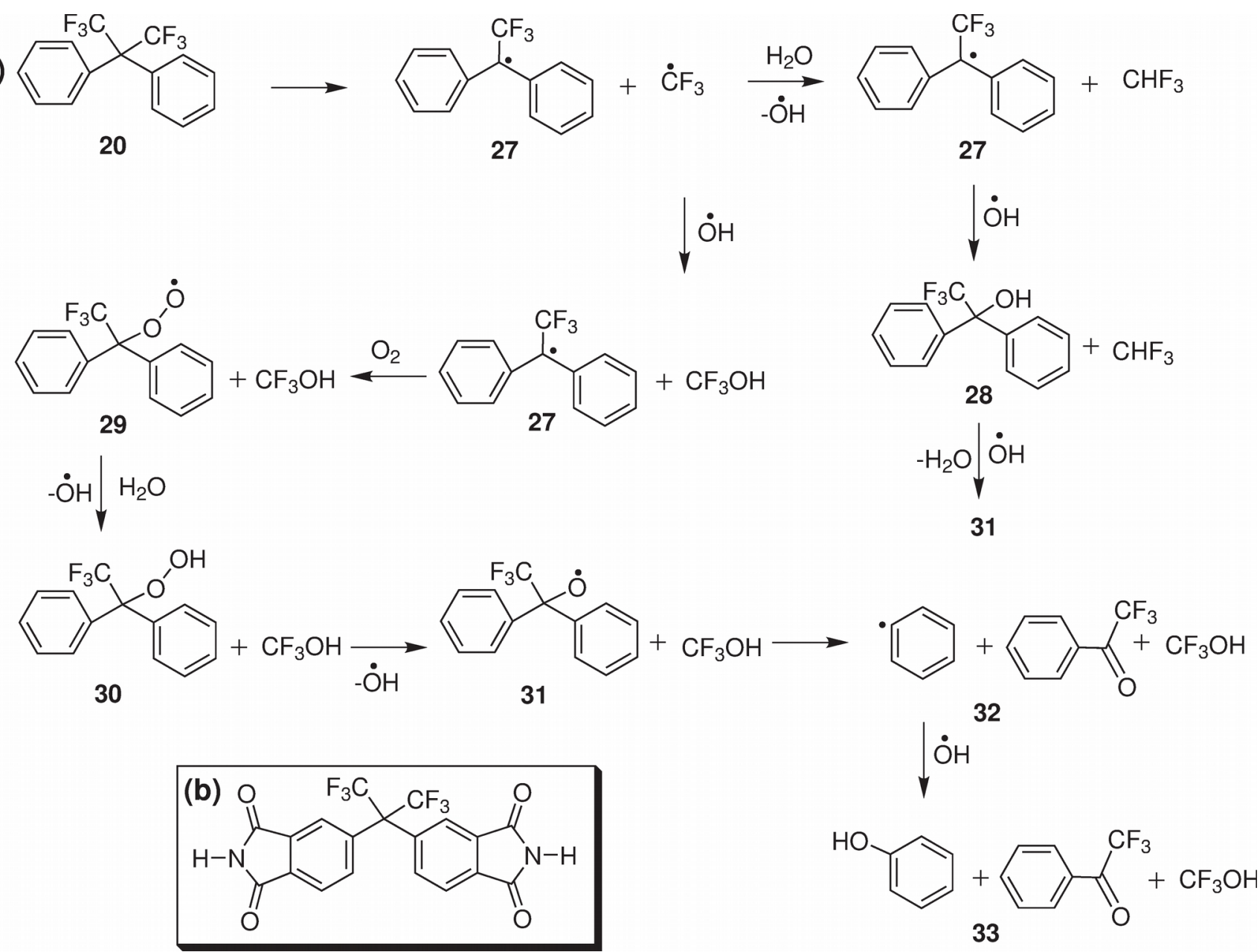

Figure S12: Oxidation routes initiated by the thermolysis of $\mathrm{C}-\mathrm{CF}_{3}$ bond for the oxidation of 6FDA. 


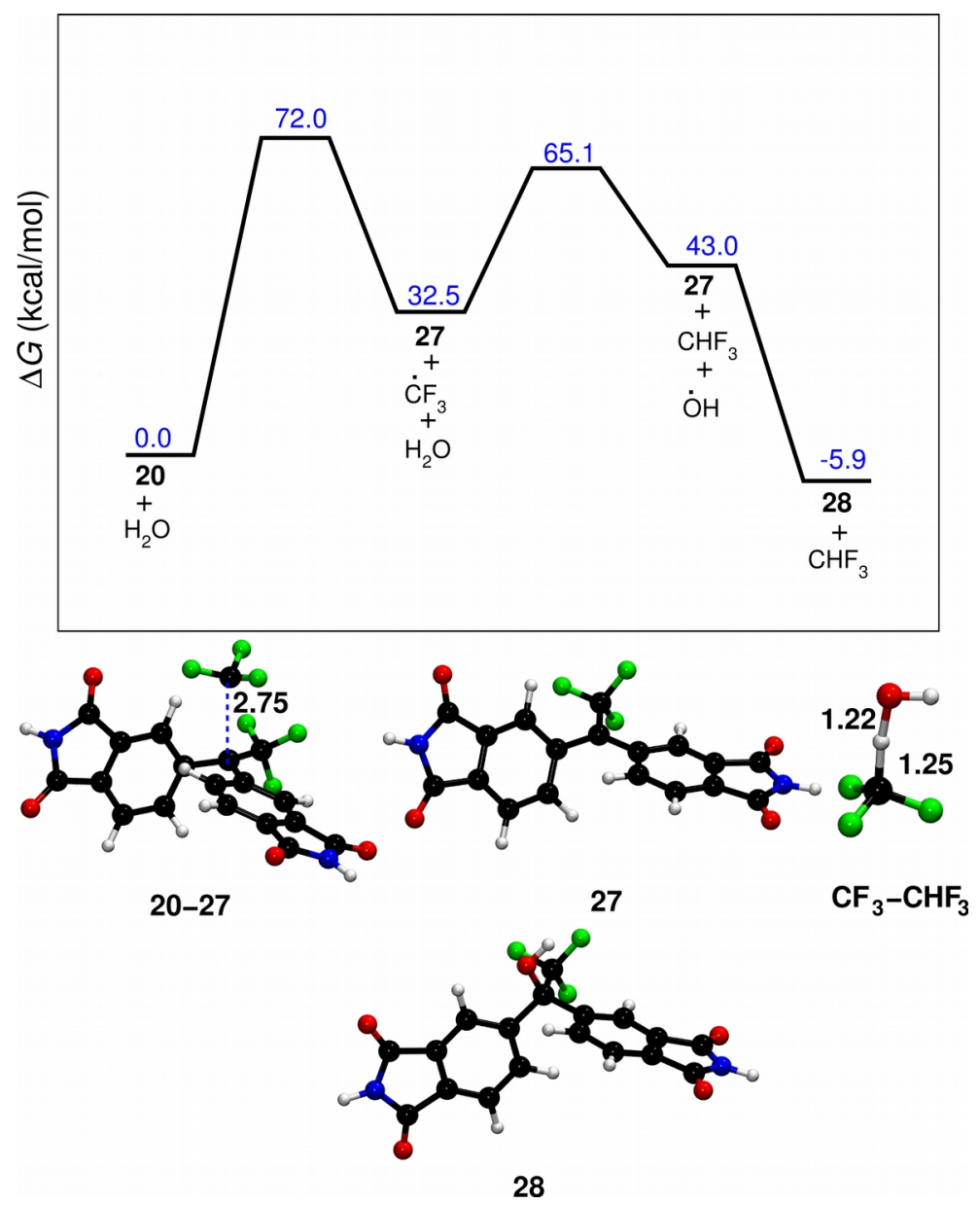

Figure S13: Free energy profile and optimized structures involve in the oxidation of 6FDA to form $\mathrm{CHF}_{3}$ and 28 along the oxidation pathway initiated by the thermolysis of $\mathrm{C}-\mathrm{CF}_{3}$ bond. 


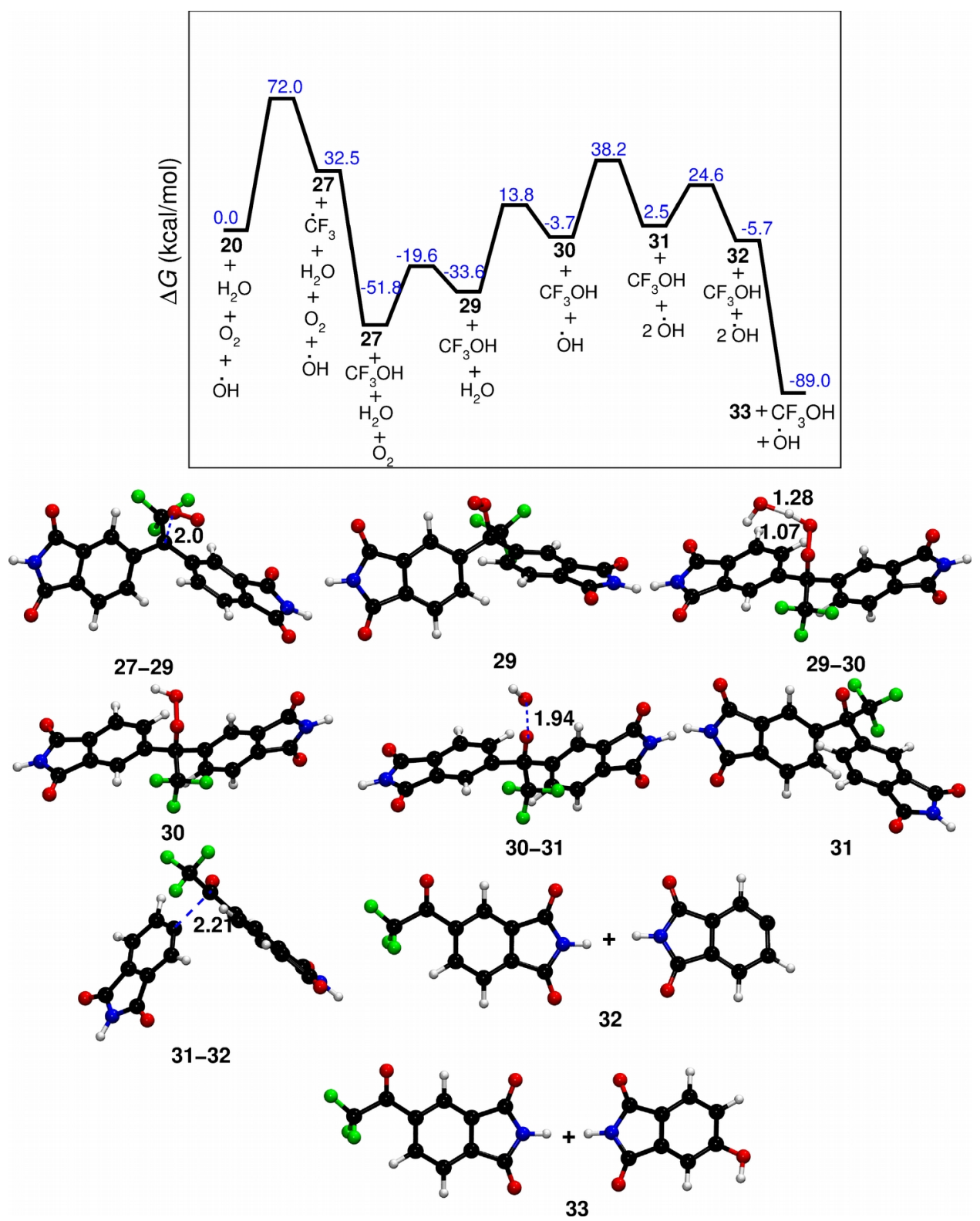

Figure S14: Free energy profile and optimized structures involve in the oxidation of $6 \mathrm{FDA}$ to form $\mathrm{CF}_{3} \mathrm{OH}$ and 33 along the oxidation pathway initiated by the thermolysis of $\mathrm{C}-\mathrm{CF}_{3}$. 


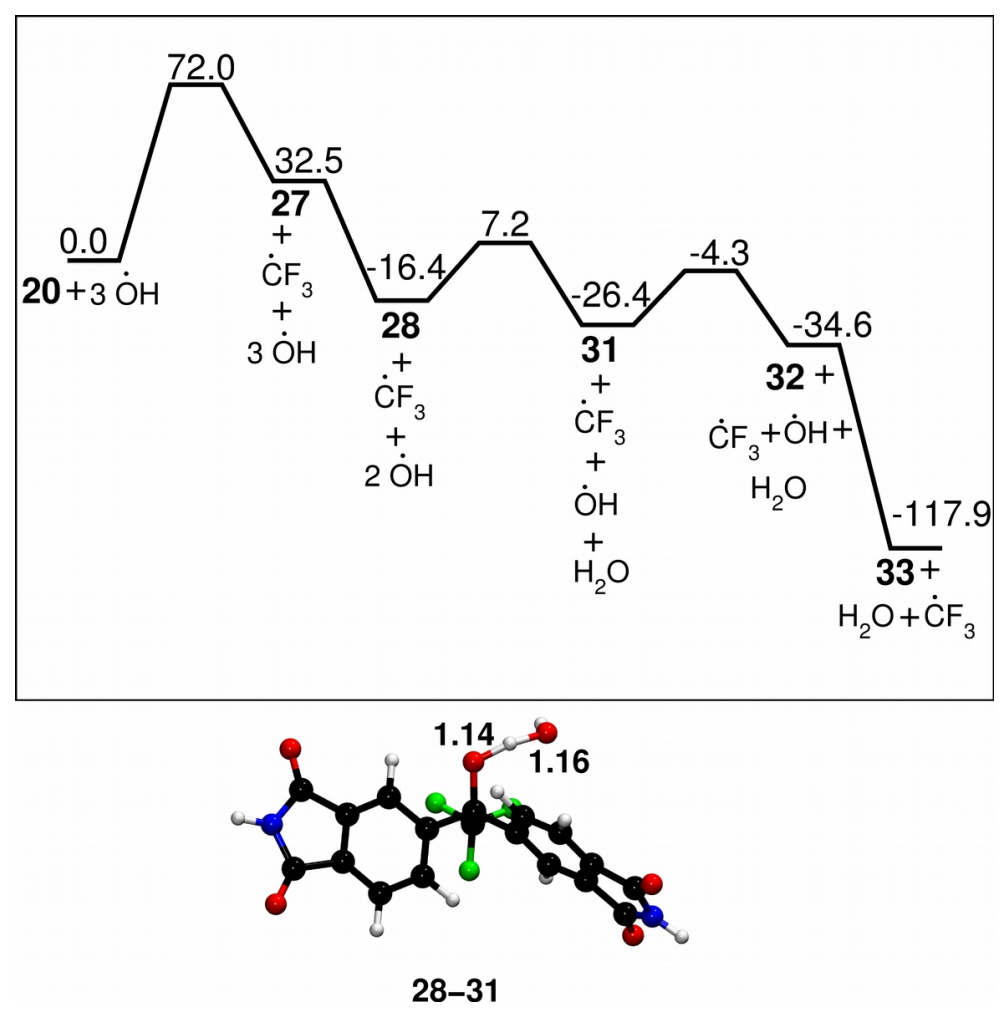

Figure S15: Free energy profile and optimized structures involve in the oxidation of 6FDA to form $\mathbf{3 3}$ and CF3 radical.

Table S7: Free energies (in au) computed by employing MPW1K/6-31+G(d,p) level of theory at $600 \mathrm{~K}$ for the oxidation of 6 FDA along routes which are initiated by the thermolysis of $\mathrm{C}-\mathrm{CF}_{3}$ bond.

\begin{tabular}{|c|c|}
\hline & Free energy (au) \\
\hline $\mathbf{2 0 - 2 7}$ & -1737.680173 \\
\hline $\mathbf{2 7}$ & -1400.229080 \\
\hline $\mathbf{2 7 - 2 9}$ & -1550.481452 \\
\hline $\mathbf{2 9}$ & -1550.503696 \\
\hline $\mathbf{2 9 - 3 0}$ & -1626.850576 \\
\hline $\mathbf{3 0}$ & -1551.136494 \\
\hline $\mathbf{2 8}$ & -1476.049023 \\
\hline $\mathbf{2 8 - 3 1}$ & -1551.753353 \\
\hline $\mathbf{3 0 - 3 1}$ & -1551.069656 \\
\hline $\mathbf{3 1}$ & -1475.384496 \\
\hline $\mathbf{3 1 - 3 2}$ & -1475.349312 \\
\hline $\mathbf{3 2}$ & $-963.153121+-$ \\
\hline
\end{tabular}




\begin{tabular}{|c|c|}
\hline 33 & $\begin{array}{c}-963.153121+- \\
588.119277\end{array}$ \\
\hline $\mathrm{CF} 3$ & -337.514058 \\
\hline $\mathrm{CF}_{3}-\mathrm{CHF}_{3}$ & -413.884574 \\
\hline $\mathrm{CHF}_{3}$ & -338.177851 \\
\hline $\mathrm{CF}_{3} \mathrm{OH}$ & -413.390453 \\
\hline
\end{tabular}

Table S8: Free energy barriers (in kcal/mol) computed by employing MPW1K/6-31+G(d,p) level of theory at $600 \mathrm{~K}$ for 6FDA oxidation initiated by the thermolysis of $\mathrm{C}_{-} \mathrm{CF}_{3}$ bond.

\begin{tabular}{|c|c|c|}
\hline \multirow{2}{*}{ Step } & \multicolumn{2}{|c|}{$\begin{array}{c}\text { Free energy barrier } \\
\text { (kcal/mol) }\end{array}$} \\
\cline { 2 - 3 } & Forward & Reverse \\
\hline $\mathbf{2 0 - 2 7}$ & 71.98 & 39.51 \\
\hline $\mathbf{2 7 - 2 9}$ & 32.20 & 13.96 \\
\hline $\mathbf{2 9 - 3 0}$ & 47.41 & 17.51 \\
\hline $\mathbf{2 7 - 2 8}$ & 0.00 & 48.91 \\
\hline $\mathbf{2 8 - 3 1}$ & 23.63 & 33.62 \\
\hline $\mathbf{3 0 - 3 1}$ & 41.94 & 35.66 \\
\hline $\mathbf{3 1 - 3 2}$ & 22.08 & 30.30 \\
\hline $\mathbf{3 2 - 3 3}$ & 0.00 & 83.34 \\
\hline $\mathrm{CF}_{3}-\mathrm{CHF}_{3}$ & 32.57 & 22.12 \\
\hline $\mathrm{CF}_{3}-\mathrm{CF}_{3} \mathrm{OH}$ & 0.00 & 84.34 \\
\hline
\end{tabular}

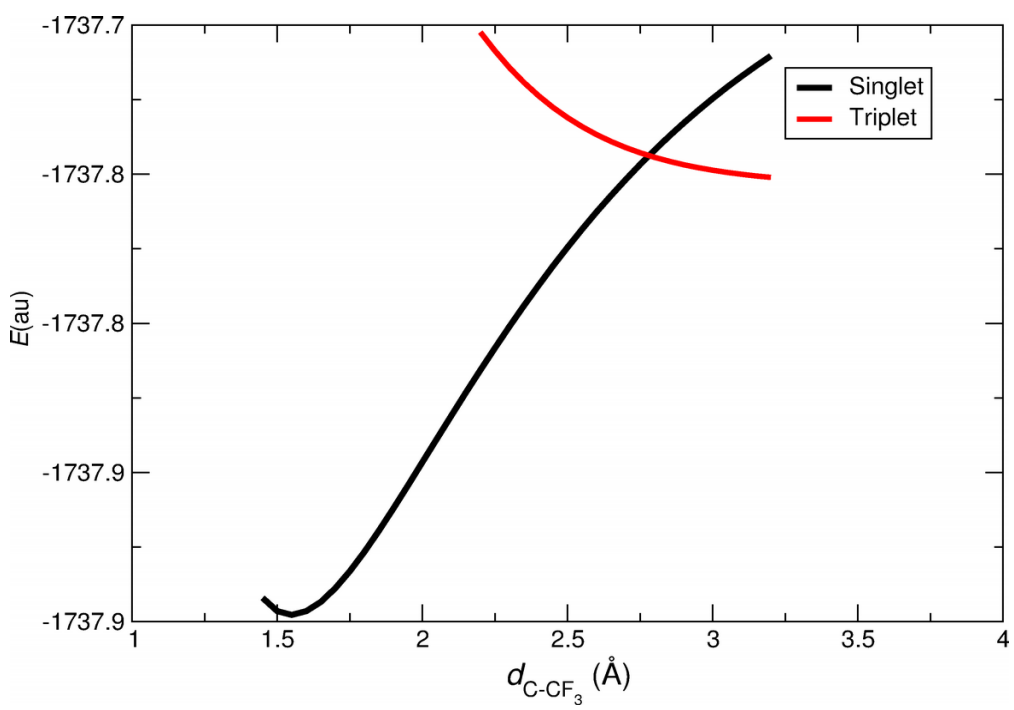

Figure S16: Singlet (black curve) and triplet (red curve) potential energy curves for the C-CF3 bond dissociation of 20 using MPW1K/6-31+G(d,p) level of theory. 


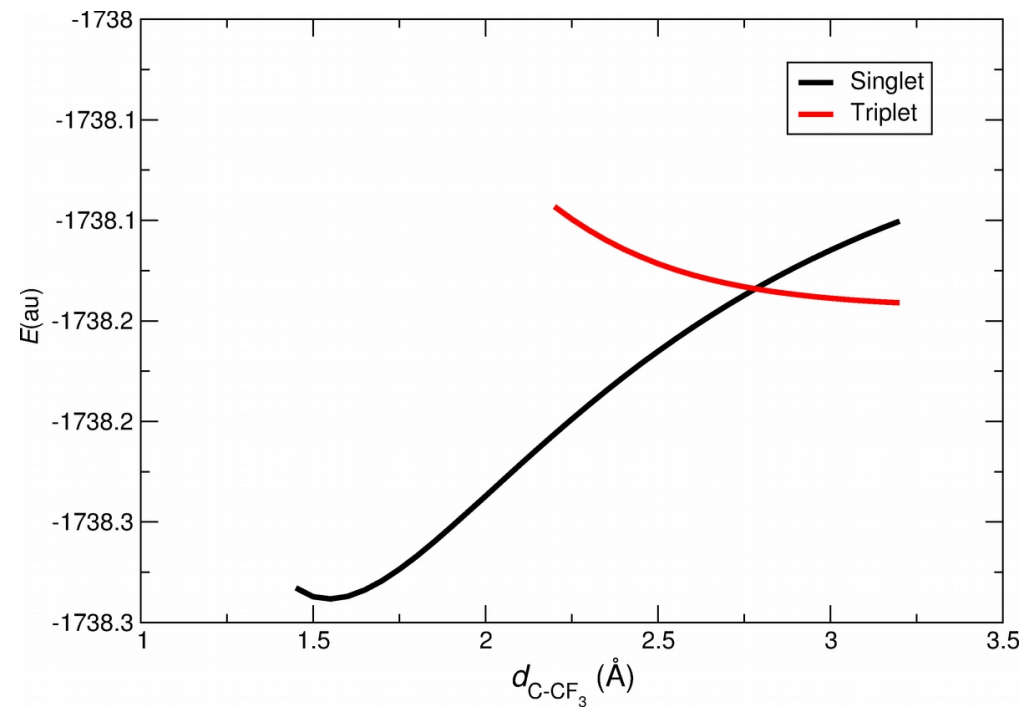

Figure S17: Singlet (black curve) and triplet (red curve) potential energy curves for the C-CF3 bond dissociation of 20 using MPW1K/6-311++G(d,p) level of theory.

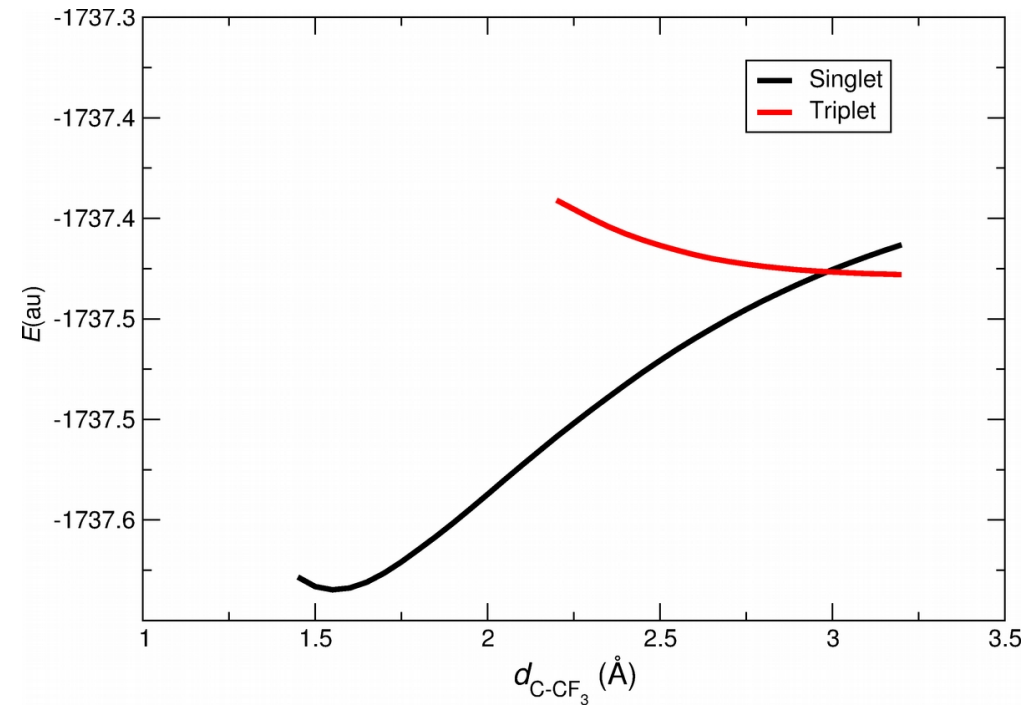

Figure S18: Singlet (black curve) and triplet (red curve) potential energy curves for the C-CF3 bond dissociation of 20 using M06/6-31+G(d,p) level of theory. 
Table S9: Total electronic energies (in au) of $\mathbf{2 0}$ and transition state of step $\mathbf{2 0} \rightarrow \mathbf{2 7}$ (20-27) using MPW1K/6-31+G(d,p), MPW1K/6-311++G(d,p) and M06/6-31+G(d,p) level of theories.

\begin{tabular}{|c|c|c|c|}
\hline & MPW1K/6-31+G(d,p) & MPW1K/6-311++G(d,p) & M06/6-31+G(d,p) \\
\hline $\mathbf{2 0}$ & -1737.89776024 & -1738.28827091 & -1737.58473264 \\
\hline $\mathbf{2 0 - 2 7}$ & -1737.77157777 & -1738.16016558 & -1737.45852422 \\
\hline
\end{tabular}

Table S10: Difference in total electronic energies (in kcal/mol) between 20 and transition state of step $20 \rightarrow \mathbf{2 7}$ (20-27) using MPW1K/6-31+G(d,p), MPW1K/6-311++G(d,p) and M06/6-31+G(d,p) level of theories.

\begin{tabular}{|c|c|c|}
\hline MPW1K/6-31+G(d,p) & MPW1K/6-311++G(d,p) & M06/6-31+G(d,p) \\
\hline 79.2 & 80.4 & 79.2 \\
\hline
\end{tabular}
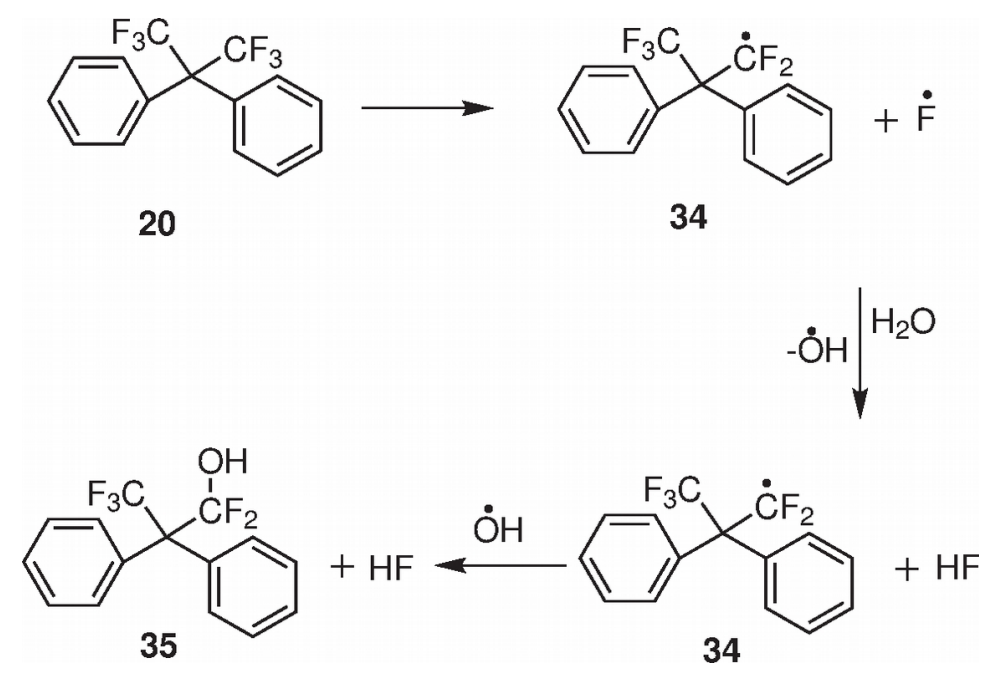

Figure S19: Oxidation routes initiated by the thermolysis of C-F bond of 6FDA. 


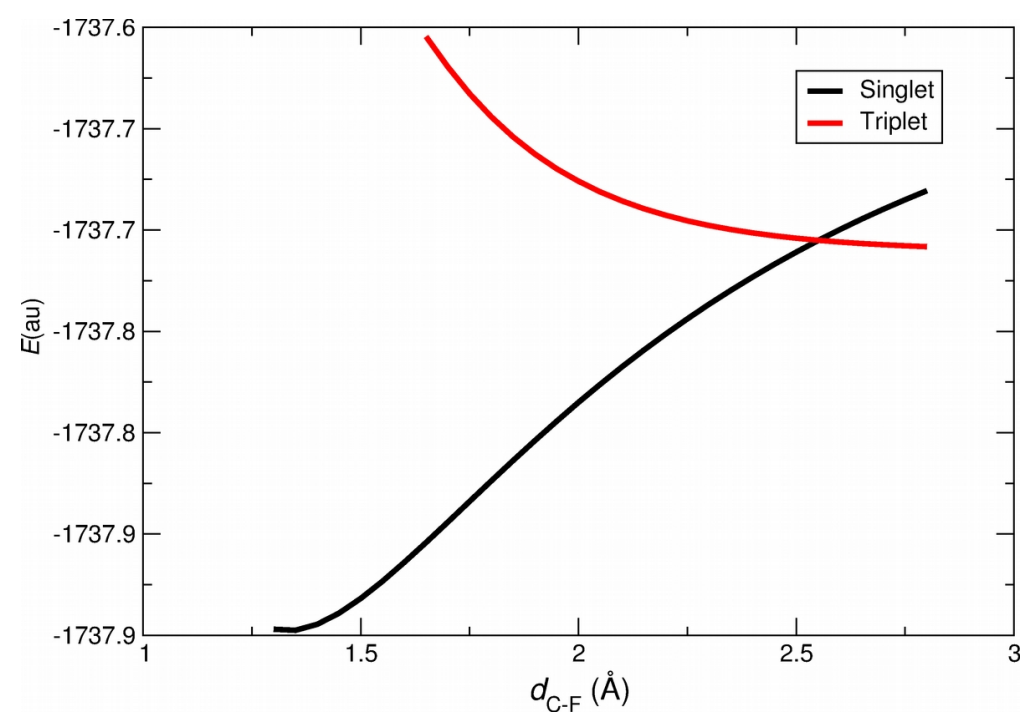

Figure S20: Singlet (black curve) and triplet (red curve) potential energy curves for the C-F bond dissociation of 20 using MPW1K/6-31+G(d,p) level of theory.

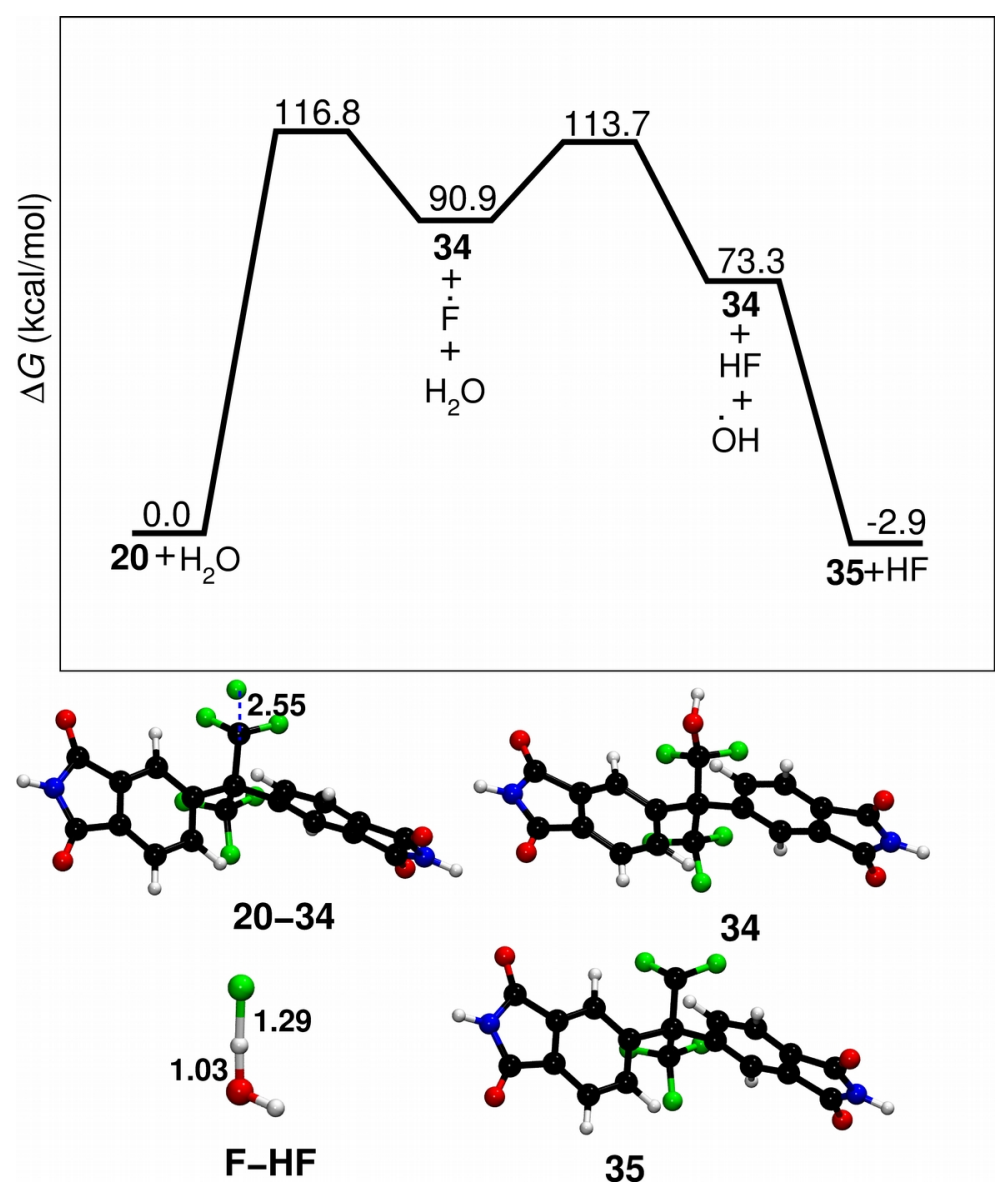

Figure S21: Free energy profile and optimized structures involve in the oxidation of 6FDA to form HF and 35 along the oxidation route which is initiated by the thermolysis of C-F bond. 
Table S11: Free energies (in au) computed by employing MPW1K/6-31+G(d,p) level of theory at $600 \mathrm{~K}$ for 6FDA oxidation route initiated by the thermolysis of $\mathrm{C}-\mathrm{F}$ bond.

\begin{tabular}{|c|c|}
\hline & Free energy (au) \\
\hline $\mathbf{2 0 - 3 4}$ & -1737.608745 \\
\hline $\mathbf{3 4}$ & -1637.914031 \\
\hline $\mathbf{3 5}$ & -1713.777375 \\
\hline $\mathbf{F}$ & -99.735956 \\
\hline F-HF & -176.121979 \\
\hline HF+OH & -100.444386 \\
\hline
\end{tabular}

Table S12: Free energy barriers (in kcal/mol) computed by employing MPW1K/6-31+G(d,p) level of theory at $600 \mathrm{~K}$ for 6FDA oxidation initiated by the thermolysis of C-F bond.

\begin{tabular}{|c|c|c|}
\hline \multirow{2}{*}{ Step } & \multicolumn{2}{|c|}{ Free energy barrier (kcal/mol) } \\
\cline { 2 - 3 } & Forward & Reverse \\
\hline $\mathbf{2 0 - 3 4}$ & 116.80 & 25.88 \\
\hline $\mathbf{3 4 - 3 5}$ & 0.00 & 76.15 \\
\hline F-HF & 22.84 & 40.41 \\
\hline
\end{tabular}


(a)

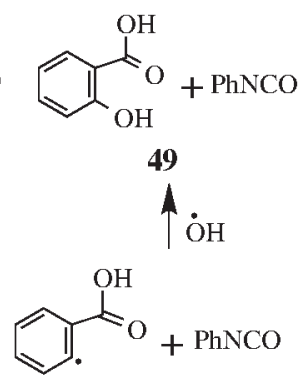

49$$
48
$$

48

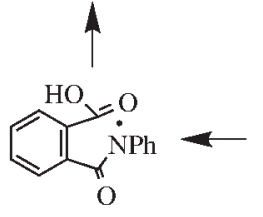

40

$\downarrow \dot{\mathrm{O}} \mathrm{H}$
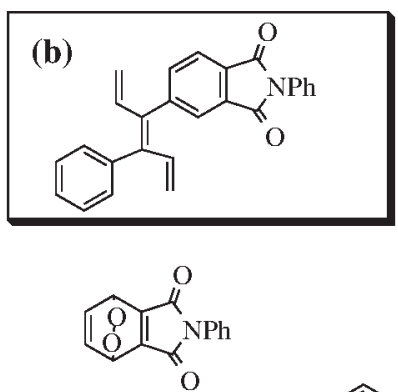

$\mathrm{O}$

Qll $+\mathrm{PhNCO}$

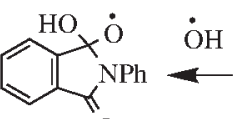

39

36 $\mathbf{3 8 b}$

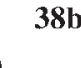

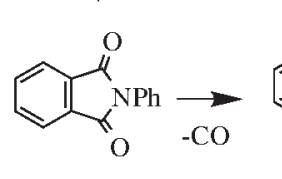

1<smiles>CNC(=O)c1ccccc1</smiles>

37

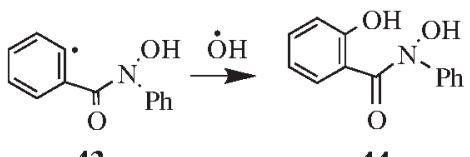

43

44

$-\mathrm{H}_{2} \mathrm{O} \downarrow \dot{\mathrm{O} H}$

$$
\underbrace{\mathrm{OH}}_{\mathrm{O}}+\mathrm{PhNO} \stackrel{\dot{\mathrm{O}}}{-}
$$

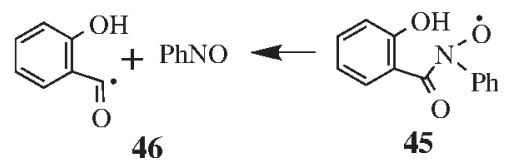

Figure S22: (a) Mechanism of oxidation of BTD component of HFPE (b) Chemical structure of $\mathbf{3 6}$ used to study step $36 \rightarrow 50$. 

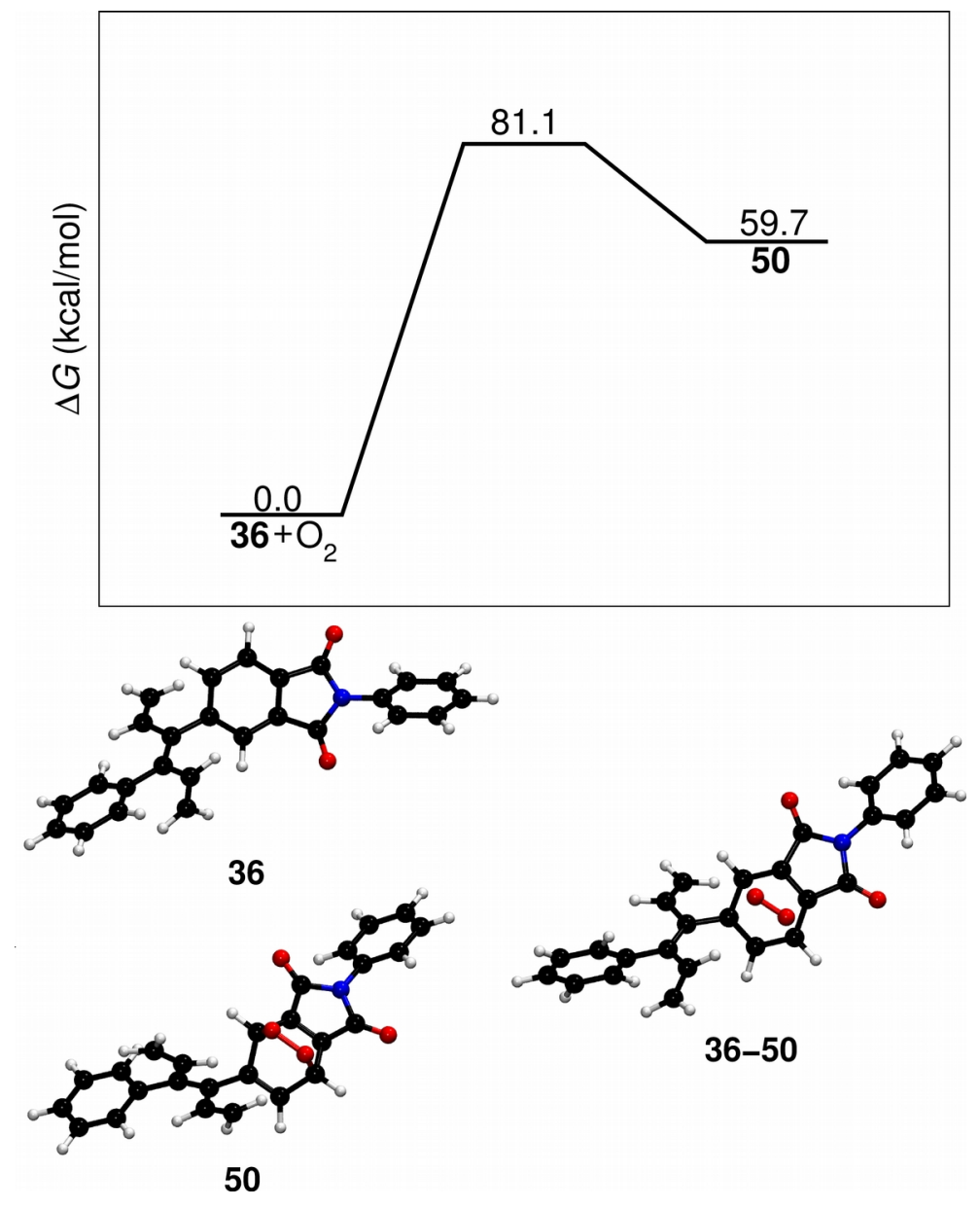

Figure S23: Free energy profile and optimized structures involve in the oxidation of BTD to form $\mathbf{5 0 .}$ 

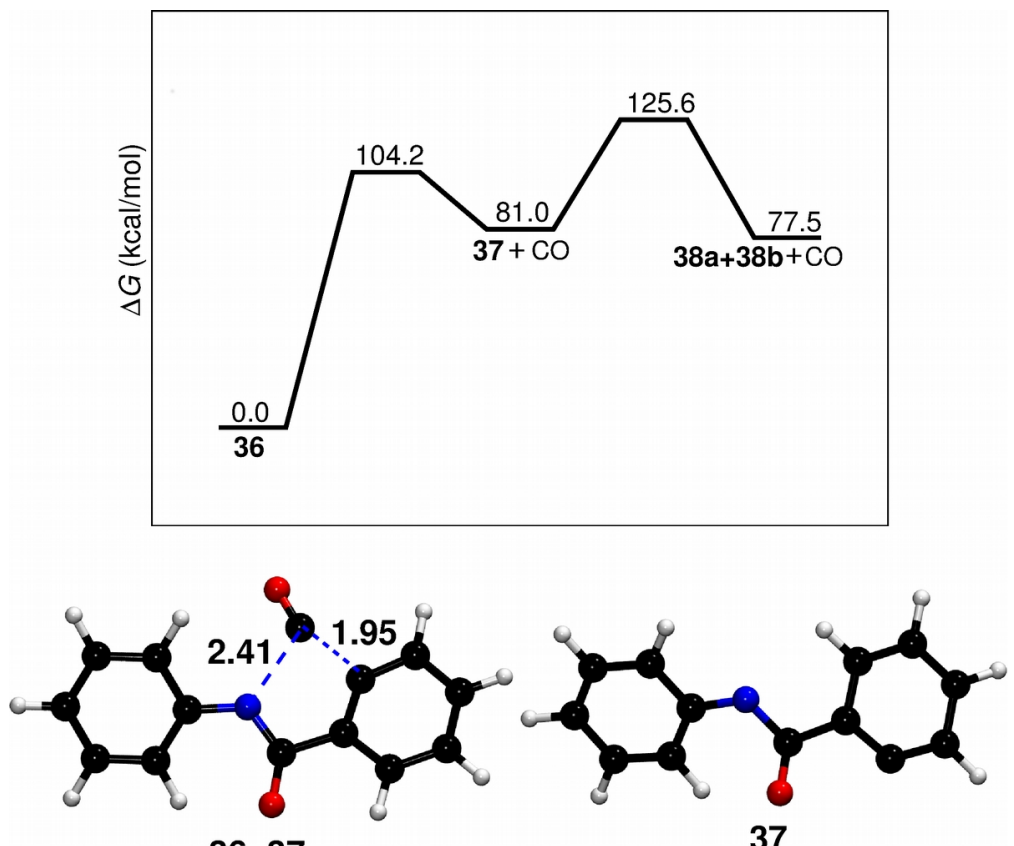

36-37

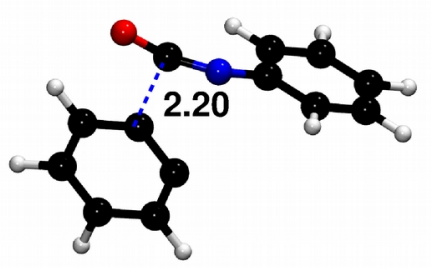

37-38

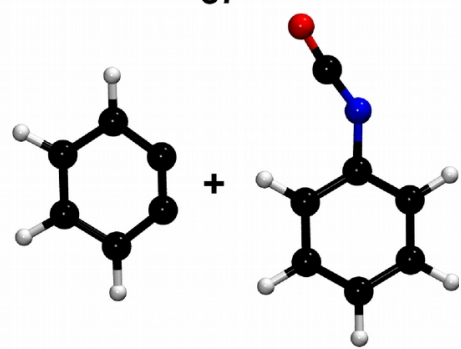

38a

38b

Figure S24: Free energy profile and optimized structures involve in the oxidation of BTD along the oxidation route initiated by the imide bond cleavage. 


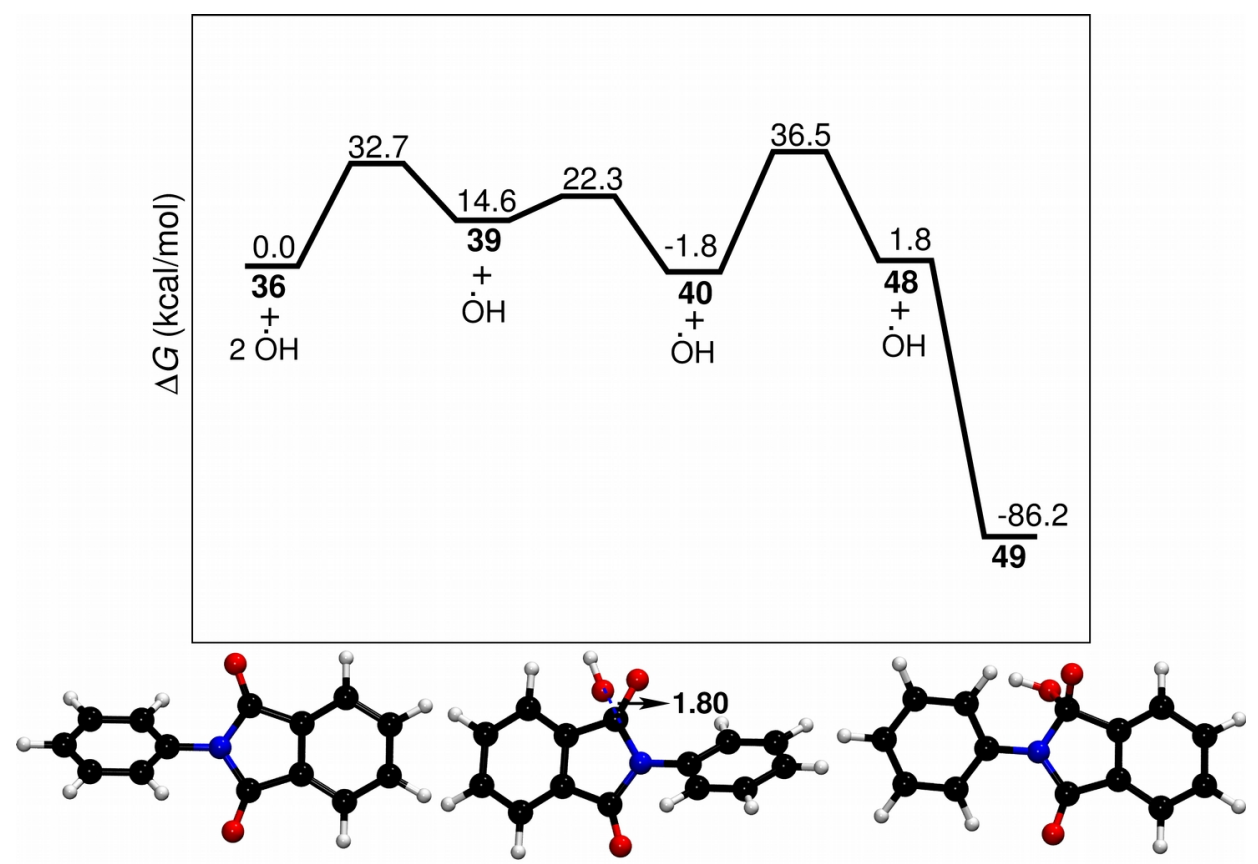

36

36-39

39
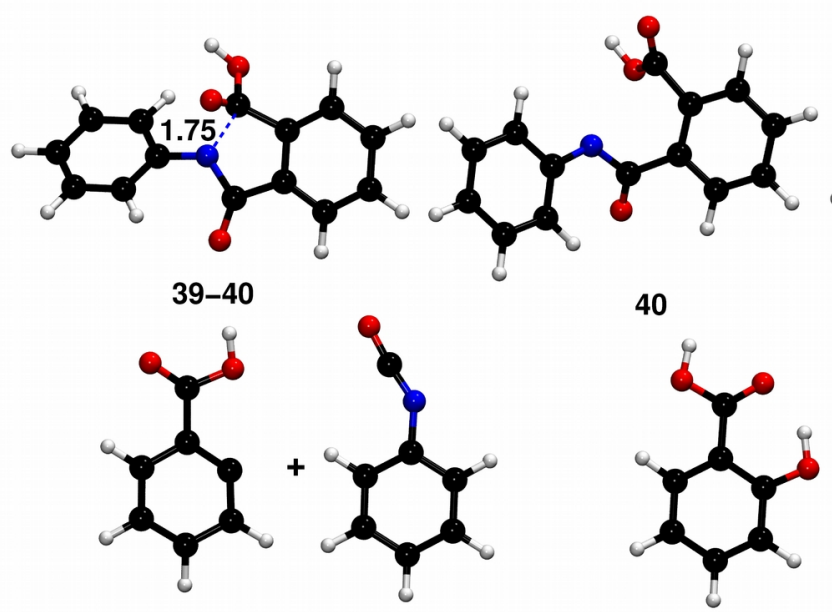

48

40
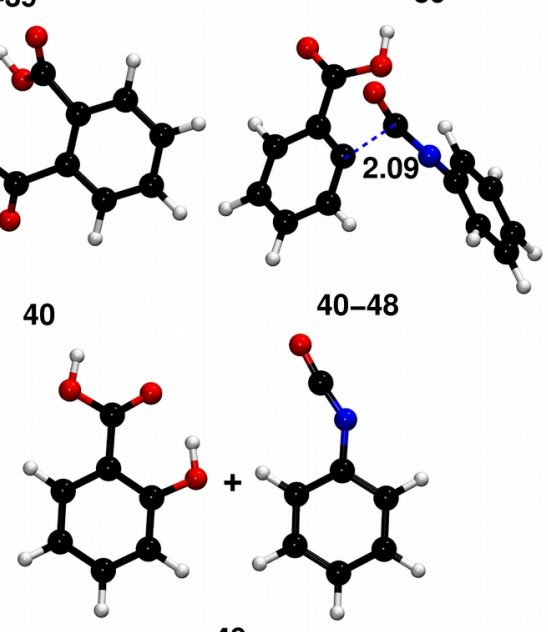

49

Figure S25: Free energy profile and optimized structures involve in the oxidation of BTD to form 49. Here the reaction is initiated by the attack of $\dot{\mathrm{O}} \mathrm{H}$ on the carbonyl $\mathrm{C}$ of the five membered ring. 
Table S13: Free energies (in au) computed by employing MPW1K/6-31+G(d p) level of theory at $600 \mathrm{~K}$ for BTD oxidation.

\begin{tabular}{|c|c|}
\hline & Free energy (au) \\
\hline 36 & -743.854518 \\
\hline$* 36 \mathbf{a}$ & -1206.874739 \\
\hline $36 a-50$ & -1357.049134 \\
\hline 50 & -1357.083252 \\
\hline $36-37$ & -743.688402 \\
\hline 37 & -630.420388 \\
\hline $37-38$ & -630.349368 \\
\hline $38 a+38 b$ & $-230.833776+-399.592283$ \\
\hline $36-39$ & -819.544442 \\
\hline 39 & -819.573316 \\
\hline $39-40$ & -819.561121 \\
\hline 40 & -819.599530 \\
\hline 41 & -895.377695 \\
\hline $41-42$ & -971.085180 \\
\hline 42 & -894.709777 \\
\hline $42-43$ & -894.690981 \\
\hline 43 & -706.198201 \\
\hline 44 & -782.067470 \\
\hline 44-45 & -857.785327 \\
\hline 45 & -781.460254 \\
\hline $45-46$ & -781.400166 \\
\hline 46 & $-361.411199+-420.002299$ \\
\hline 47 & $-361.411199+-495.883617$ \\
\hline $40-48$ & -819.538471 \\
\hline 48 & $-420.001470+-399.592281$ \\
\hline 49 & $-399.592281+-495.883617$ \\
\hline CO & -113.304953 \\
\hline $\mathrm{CO} 2$ & -188.546181 \\
\hline
\end{tabular}

*36a was used for step $\mathbf{3 6} \rightarrow \mathbf{5 0}$. For all other steps $\mathbf{3 6}$ was used 
Table S14: Free energy barriers (in $\mathrm{kcal} / \mathrm{mol}$ ) computed by employing MPW1K/6-31+G(d,p) level of theory at $600 \mathrm{~K}$ for BTD oxidation.

\begin{tabular}{|c|c|c|}
\hline \multirow{2}{*}{ Step } & \multicolumn{2}{|c|}{ Free energy } \\
\cline { 2 - 3 } & Forward & Reverse \\
\hline *36-50 & 81.13 & 21.41 \\
\hline $\mathbf{3 6 - 3 7}$ & 104.24 & 23.18 \\
\hline $\mathbf{3 7 - ( 3 8 a + 3 8 b )}$ & 44.57 & 48.12 \\
\hline $\mathbf{3 6 - 3 9}$ & 32.67 & 18.12 \\
\hline $\mathbf{3 9 - 4 0}$ & 7.65 & 24.10 \\
\hline $\mathbf{4 0 - 4 1}$ & 0.00 & 22.70 \\
\hline $\mathbf{4 1 - 4 2}$ & 21.65 & 29.51 \\
\hline $\mathbf{4 2 - 4 3}$ & 11.79 & 33.51 \\
\hline $\mathbf{4 3 - 4 4}$ & 0.00 & 79.87 \\
\hline $\mathbf{4 4 - 4 5}$ & 15.14 & 61.09 \\
\hline $\mathbf{4 5 - 4 6}$ & 37.71 & 8.37 \\
\hline $\mathbf{4 6 - 4 7}$ & 0.00 & 87.43 \\
\hline $\mathbf{4 0 - 4 8}$ & 38.31 & 34.69 \\
\hline $\mathbf{4 8 - 4 9}$ & 0.00 & 87.95 \\
\hline
\end{tabular}

${ }^{*} \mathbf{3 6 a}$ was used to compute the free energy barrier for step $\mathbf{3 6} \rightarrow \mathbf{5 0}$ 


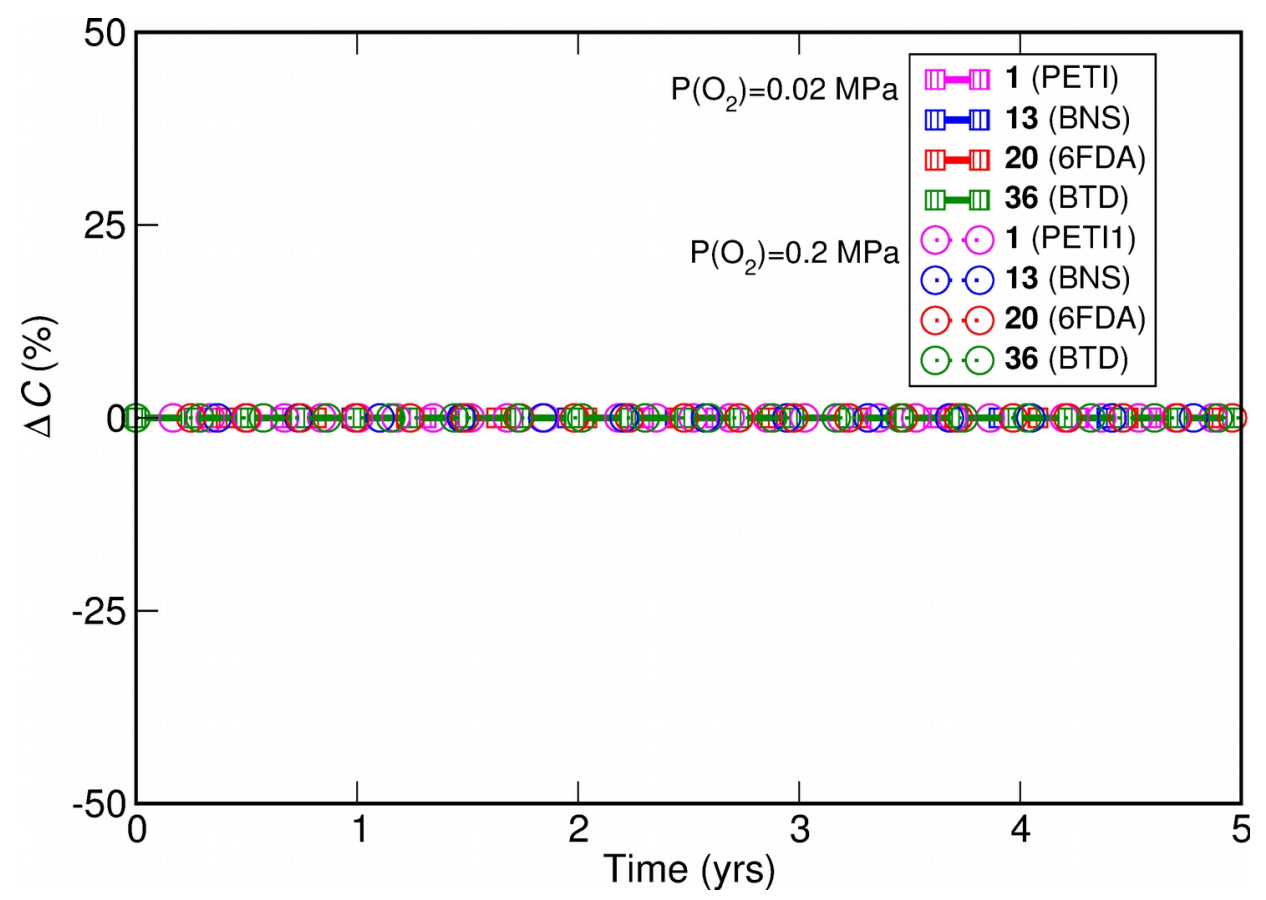

Figure S26 Percentage of decrease in concentration of different components of HFPE at $600 \mathrm{~K}$ for $\mathrm{O}_{2}$ concentration ten times that of normal atmospheric concentration (0.02 MPa). Here, $\Delta C=\frac{C(t)-C(0)}{C(0)}$

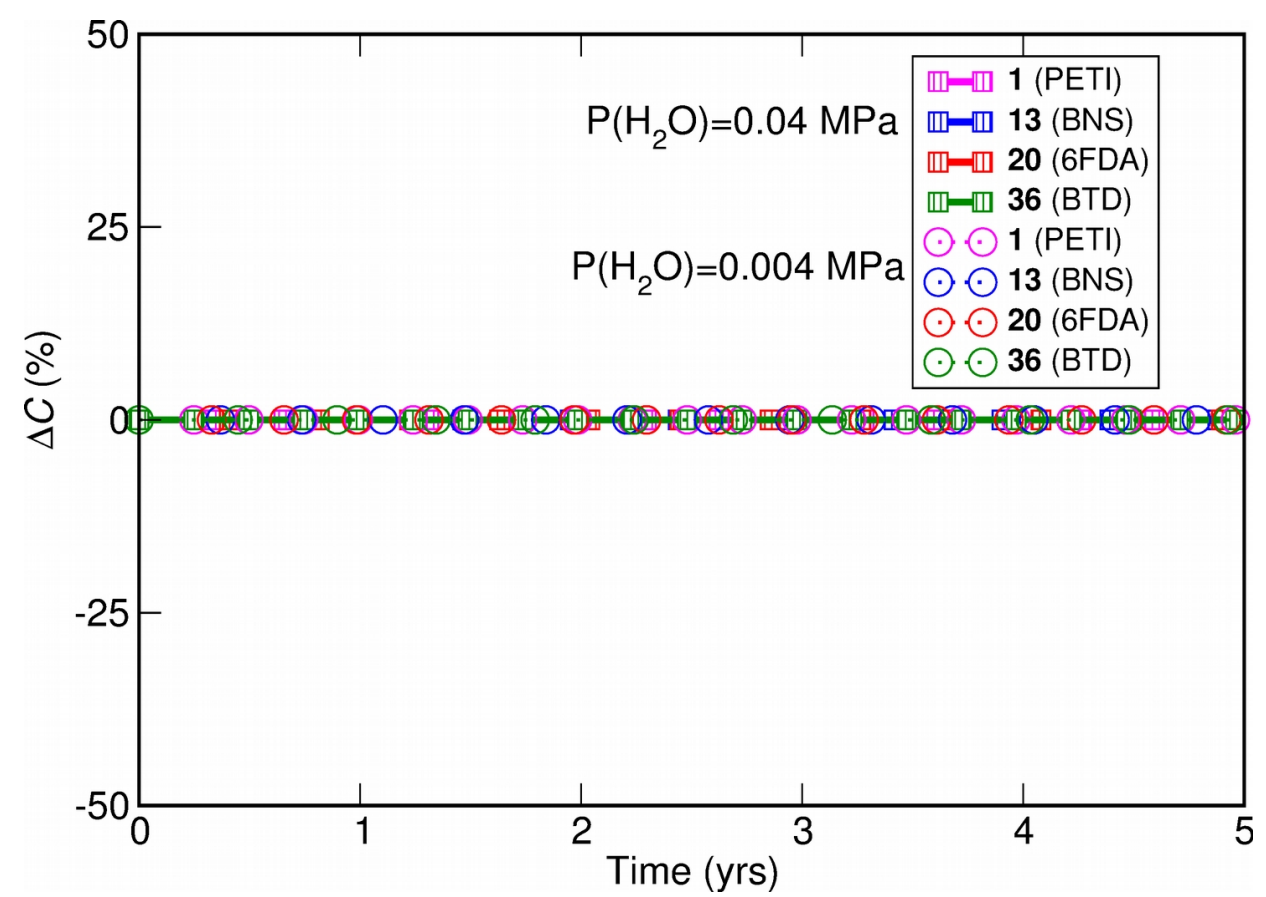

Figure S27: Percentage of decrease in concentration of different components of HFPE at $600 \mathrm{~K}$ for $\mathrm{H}_{2} \mathrm{O}$ concentration ten times that of normal atmospheric concentration (0.004 MPa). 
Table S15: Free energy barriers and corresponding rate constants computed at $600 \mathrm{~K}$ for all the elementary steps studied for the HFPE degradation.

\begin{tabular}{|c|c|c|c|c|}
\hline \multirow[t]{2}{*}{ Step } & \multicolumn{2}{|c|}{ Free energy barrier (kcal/mol) } & \multicolumn{2}{|c|}{ Rate Constant (year ${ }^{-1}$ or L mol ${ }^{-1}$ year $\left.^{-1}\right)^{*}$} \\
\hline & Forward & Reverse & Forward & Reverse \\
\hline $1-2$ & 60.54 & 14.21 & 0.03 & $2.63 \times 10^{15}$ \\
\hline $2-4$ & 58.85 & 18.64 & 0.14 & $6.39 \times 10^{13}$ \\
\hline 4-3 & 15.83 & 50.44 & $6.75 \times 10^{14}$ & 166.79 \\
\hline $4-5$ & 0.00 & 37.82 & $3.94 \times 10^{20}$ & $6.59 \times 10^{6}$ \\
\hline $5-6$ & 38.19 & 38.17 & $4.83 \times 10^{6}$ & $4.92 \times 10^{6}$ \\
\hline $6-(7+8)$ & 3.79 & 74.55 & $1.64 \times 10^{19}$ & $2.75 \times 10^{-7}$ \\
\hline 8-9a & 0.00 & 32.96 & $3.94 \times 10^{20}$ & $3.89 \times 10^{8}$ \\
\hline 8-9b & 0.00 & 41.72 & $3.94 \times 10^{20}$ & 250349.00 \\
\hline 1-10 & 110.93 & 77.90 & $1.54 \times 10^{-20}$ & $1.66 \times 10^{-8}$ \\
\hline $1-11$ & 27.78 & 32.67 & $2.99 \times 10^{10}$ & $4.96 \times 10^{8}$ \\
\hline $11-12$ & 0.00 & 39.83 & $3.94 \times 10^{20}$ & $1.22 \times 10^{6}$ \\
\hline $12-6$ & 17.51 & 37.38 & $1.65 \times 10^{14}$ & $9.54 \times 10^{6}$ \\
\hline $13-15$ & 59.78 & 0.99 & 0.07 & $1.72 \times 10^{20}$ \\
\hline 13a-14 & 77.73 & 27.32 & $1.91 \times 10^{-8}$ & $4.40 \times 10^{10}$ \\
\hline $15-16$ & 50.56 & 17.56 & 150.82 & $1.58 \times 10^{14}$ \\
\hline $16-17$ & 23.31 & 40.91 & $1.27 \times 10^{12}$ & 493853.00 \\
\hline $13-18$ & 19.69 & 16.62 & $2.65 \times 10^{13}$ & $3.48 \times 10^{14}$ \\
\hline 18-19 & 0.00 & 42.28 & $3.94 \times 10^{20}$ & 156516.00 \\
\hline $20-21$ & 82.05 & 24.93 & $5.10 \times 10^{-10}$ & $3.27 \times 10^{11}$ \\
\hline $20-22$ & 62.65 & 1.84 & 0.01 & $8.42 \times 10^{19}$ \\
\hline $22-23$ & 51.47 & 19.34 & 70.30 & $3.55 \times 10^{13}$ \\
\hline $23-24$ & 24.03 & 43.30 & $6.96 \times 10^{11}$ & 66528.9 \\
\hline $20-25$ & 23.69 & 22.19 & $9.25 \times 10^{11}$ & $3.26 \times 10^{12}$ \\
\hline $25-26$ & 0.00 & 37.87 & $3.94 \times 10^{20}$ & $6.32 \times 10^{6}$ \\
\hline $20-27$ & 71.98 & 39.51 & $2.38 \times 10^{-6}$ & $1.60 \times 10^{6}$ \\
\hline $27-29$ & 32.20 & 13.96 & $7.35 \times 10^{8}$ & $3.24 \times 10^{15}$ \\
\hline $29-30$ & 47.41 & 17.51 & 2117.83 & $1.65 \times 10^{14}$ \\
\hline $27-28$ & 0.00 & 48.91 & $3.94 \times 10^{20}$ & 601.86 \\
\hline
\end{tabular}




\begin{tabular}{|c|c|c|c|c|}
\hline 28-31 & 23.63 & 33.62 & $9.73 \times 10^{11}$ & $2.23 \times 10^{8}$ \\
\hline $30-31$ & 41.94 & 35.66 & 208165.00 & $4.04 \times 10^{7}$ \\
\hline $31-32$ & 22.08 & 30.30 & $3.57 \times 10^{12}$ & $3.62 \times 10^{9}$ \\
\hline $32-33$ & 0.00 & 83.34 & $3.94 \times 10^{20}$ & $1.73 \times 10^{-10}$ \\
\hline $\mathrm{CF}_{3}-\mathrm{CHF}_{3}$ & 32.57 & 22.12 & $5.39 \times 10^{8}$ & $3.45 \times 10^{12}$ \\
\hline $\mathrm{CF}_{3}-\mathrm{CF}_{3} \mathrm{OH}$ & 0.00 & 84.34 & $3.94 \times 10^{20}$ & $7.48 \times 10^{-11}$ \\
\hline 20-34 & 116.80 & 25.88 & $1.12 \times 10^{-22}$ & $1.47 \times 10^{11}$ \\
\hline 34-35 & 0.00 & 76.15 & $3.94 \times 10^{20}$ & $7.19 \times 10^{-8}$ \\
\hline F-HF & 22.84 & 40.41 & $1.89 \times 10^{12}$ & 751155.00 \\
\hline $36-37$ & 104.24 & 23.18 & $4.22 \times 10^{-18}$ & $1.42 \times 10^{12}$ \\
\hline $\begin{array}{c}37- \\
(38 a+38 b)\end{array}$ & 44.57 & 48.12 & 22929.60 & 1167.52 \\
\hline $36-39$ & 32.67 & 18.12 & $4.96 \times 10^{8}$ & $9.89 \times 10^{13}$ \\
\hline $39-40$ & 7.65 & 24.10 & $6.44 \times 10^{17}$ & $6.56 \times 10^{11}$ \\
\hline $40-41$ & 0.00 & 22.70 & $3.94 \times 10^{20}$ & $2.12 \times 10^{12}$ \\
\hline $41-42$ & 21.65 & 29.51 & $5.12 \times 10^{12}$ & $7.02 \times 10^{9}$ \\
\hline $42-43$ & 11.79 & 33.51 & $2.00 \times 10^{16}$ & $2.45 \times 10^{8}$ \\
\hline $43-44$ & 0.00 & 79.87 & $3.94 \times 10^{20}$ & $3.18 \times 10^{-9}$ \\
\hline $44-45$ & 15.14 & 61.09 & $1.20 \times 10^{15}$ & 0.02 \\
\hline $45-46$ & 37.71 & 8.37 & $7.23 \times 10^{6}$ & $3.52 \times 10^{17}$ \\
\hline $46-47$ & 0.00 & 87.43 & $3.94 \times 10^{20}$ & $5.60 \times 10^{-12}$ \\
\hline $40-48$ & 38.31 & 34.69 & $4.37 \times 10^{6}$ & $9.11 \times 10^{7}$ \\
\hline $48-49$ & 0.00 & 87.95 & $3.94 \times 10^{20}$ & $3.62 \times 10^{-12}$ \\
\hline $36-50$ & 81.13 & 21.41 & $1.10 \times 10^{-9}$ & $6.26 \times 10^{12}$ \\
\hline
\end{tabular}

${ }^{*}$ The unit of rate constant is year ${ }^{-1}$ for first order reactions and $\mathrm{L} \mathrm{mol}^{-1}$ year ${ }^{-1}$ for second order reactions.

Table S16: Rate constants computed at $700 \mathrm{~K}$ for all the elementary steps studied for the HFPE degradation. Please note that the free energy barrier used here is obtained from computations done at $600 \mathrm{~K}$.

\begin{tabular}{|c|c|c|}
\hline \multirow{2}{*}{ Step } & \multicolumn{2}{|c|}{ Rate Constant $\left(\right.$ year $^{-1}$ or $\mathbf{~}$ mol $^{\mathbf{1}}$ year $^{-\mathbf{1}}$ ) } \\
\cline { 2 - 3 } & Forward & Reverse \\
\hline $\mathbf{1 - 2}$ & 57.60 & $1.68 \times 10^{16}$ \\
\hline $\mathbf{2 - 4}$ & 194.13 & $6.96 \times 10^{14}$ \\
\hline $\mathbf{4 - 3}$ & $5.25 \times 10^{15}$ & 82015.20 \\
\hline $\mathbf{4 - 5}$ & $4.60 \times 10^{20}$ & $7.15 \times 10^{8}$ \\
\hline
\end{tabular}




\begin{tabular}{|c|c|c|}
\hline $5-6$ & $5.48 \times 10^{8}$ & $5.56 \times 10^{8}$ \\
\hline $6-(7+8)$ & $3.01 \times 10^{19}$ & $0.2 \times 10^{-2}$ \\
\hline 8-9a & $4.60 \times 10^{20}$ & $2.60 \times 35^{10}$ \\
\hline 8-9b & $4.60 \times 10^{20}$ & $4.33 \times 10^{7}$ \\
\hline $1-10$ & $1.07 \times 10^{-14}$ & $0.22 \times 10^{-3}$ \\
\hline 1-11 & $9.75 \times 10^{11}$ & $2.90 \times 10^{10}$ \\
\hline 11-12 & $4.60 \times 10^{20}$ & $1.69 \times 10^{8}$ \\
\hline $12-6$ & $1.57 \times 10^{15}$ & $9.81 \times 10^{8}$ \\
\hline 13-15 & 99.47 & $2.26 \times 10^{20}$ \\
\hline 13a-14 & $0.25 \times 10^{-3}$ & $1.36 \times 10^{12}$ \\
\hline $15-16$ & 75236.20 & $1.51 \times 10^{15}$ \\
\hline $16-17$ & $2.42 \times 10^{13}$ & $7.75 \times 10^{7}$ \\
\hline 13-18 & $3.27 \times 10^{14}$ & $2.97 \times 10^{15}$ \\
\hline 18-19 & $4.60 \times 10^{20}$ & $2.90 \times 10^{7}$ \\
\hline $20-21$ & $0.11 \times 10^{-4}$ & $7.56 \times 10^{12}$ \\
\hline 20-22 & 12.64 & $1.22 \times 10^{20}$ \\
\hline $22-23$ & 39111.30 & $4.21 \times 10^{14}$ \\
\hline 23-24 & $1.44 \times 10^{13}$ & $1.39 \times 10^{7}$ \\
\hline 20-25 & $1.84 \times 10^{13}$ & $5.42 \times 10^{13}$ \\
\hline $25-26$ & $4.60 \times 10^{20}$ & $6.90 \times 10^{8}$ \\
\hline $20-27$ & 0.02 & $2.12 \times 10^{8}$ \\
\hline $27-29$ & $4.06 \times 10^{10}$ & $2.01 \times 10^{16}$ \\
\hline $29-30$ & 724330.00 & $1.57 \times 10^{15}$ \\
\hline $27-28$ & $4.60 \times 10^{20}$ & 246376.00 \\
\hline 28-31 & $1.93 \times 10^{13}$ & $1.46 \times 10^{10}$ \\
\hline 30-31 & $3.70 \times 10^{7}$ & $3.38 \times 10^{9}$ \\
\hline 31-32 & $5.87 \times 10^{13}$ & $1.59 \times 10^{11}$ \\
\hline $32-33$ & $4.60 \times 10^{20}$ & $4.380 \times 10^{-6}$ \\
\hline $\mathrm{CF}_{3}-\mathrm{CHF}_{3}$ & $3.11 \times 10^{10}$ & $5.70 \times 10^{13}$ \\
\hline $\mathrm{CF}_{3}-\mathrm{CF}_{3} \mathrm{OH}$ & $4.60 \times 10^{20}$ & $2.13 \times 10^{-6}$ \\
\hline $20-34$ & $1.57 \times 10^{-17}$ & $3.57 \times 82^{12}$ \\
\hline 34-35 & $4.60 \times 10^{20}$ & $0.77 \times 10^{-3}$ \\
\hline
\end{tabular}




\begin{tabular}{|c|c|c|}
\hline F-HF & $3.40 \times 10^{13}$ & $1.11 \times 10^{8}$ \\
\hline $\mathbf{3 6 - 3 7}$ & $1.31 \times 10^{-12}$ & $2.66 \times 10^{13}$ \\
\hline $\mathbf{3 7 - ( 3 8 a + 3 8 b )}$ & $5.58 \times 10^{6}$ & 434767.00 \\
\hline $\mathbf{3 6 - 3 9}$ & $2.90 \times 10^{10}$ & $1.01 \times 10^{15}$ \\
\hline $\mathbf{3 9 - 4 0}$ & $1.88 \times 10^{18}$ & $1.37 \times 10^{13}$ \\
\hline $\mathbf{4 0 - 4 1}$ & $4.60 \times 10^{20}$ & $3.76 \times 10^{13}$ \\
\hline $\mathbf{4 1 - 4 2}$ & $8.00 \times 10^{13}$ & $2.81 \times 10^{11}$ \\
\hline $\mathbf{4 2 - 4 3}$ & $9.58 \times 10^{16}$ & $1.58 \times 10^{10}$ \\
\hline $\mathbf{4 3 - 4 4}$ & $4.60 \times 10^{20}$ & $0.53 \times 10^{-4}$ \\
\hline $\mathbf{4 4 - 4 5}$ & $8.62 \times 10^{15}$ & 38.79 \\
\hline $\mathbf{4 5 - 4 6}$ & $7.74 \times 10^{8}$ & $1.12 \times 10^{18}$ \\
\hline $\mathbf{4 6 - 4 7}$ & $4.60 \times 10^{20}$ & $2.32 \times 10^{-7}$ \\
\hline $\mathbf{4 0 - 4 8}$ & $5.03 \times 10^{8}$ & $6.78 \times 10^{9}$ \\
\hline $\mathbf{4 8 - 4 9}$ & $4.60 \times 10^{20}$ & $1.59 \times 10^{-7}$ \\
\hline $\mathbf{3 6 - 5 0}$ & $2.15 \times 10^{-5}$ & $9.50 \times 10^{13}$ \\
\hline
\end{tabular}

Table S17: Rate constants computed at $800 \mathrm{~K}$ for all the elementary steps studied for the HFPE degradation. Please note that the free energy barrier used here is obtained from computations done at $600 \mathrm{~K}$.

\begin{tabular}{|c|c|c|}
\hline \multirow[t]{2}{*}{ Step } & \multicolumn{2}{|c|}{ Rate Constant (year ${ }^{-1}$ or $\mathrm{L} \mathrm{mol}^{-1}$ year $^{-1}$ ) } \\
\hline & Forward & Reverse \\
\hline $1-2$ & 15176.6 & $6.89 \times 10^{16}$ \\
\hline $2-4$ & 43941.90 & $4.25 \times 10^{15}$ \\
\hline $4-3$ & $2.49 \times 10^{16}$ & $8.72 \times 10^{6}$ \\
\hline $4-5$ & $5.25 \times 10^{20}$ & $2.44 \times 10^{10}$ \\
\hline $5-6$ & $1.94 \times 10^{10}$ & $1.96 \times 10^{10}$ \\
\hline $6-(7+8)$ & $4.84 \times 10^{19}$ & 2.26 \\
\hline 8-9a & $5.25 \times 10^{20}$ & $5.20 \times 10^{11}$ \\
\hline 8-9b & $5.25 \times 10^{20}$ & $2.10 \times 10^{9}$ \\
\hline $1-10$ & $2.60 \times 10^{-10}$ & 0.27 \\
\hline $1-11$ & $1.35 \times 10^{13}$ & $6.24 \times 10^{11}$ \\
\hline $11-12$ & $5.25 \times 10^{20}$ & $6.90 \times 10^{9}$ \\
\hline $12-6$ & $8.65 \times 10^{15}$ & $3.22 \times 10^{10}$ \\
\hline $13-15$ & 24479.70 & $2.82 \times 10^{20}$ \\
\hline
\end{tabular}




\begin{tabular}{|c|c|c|}
\hline 13a-14 & 0.31 & $1.81 \times 10^{13}$ \\
\hline $15-16$ & $8.09 \times 10^{6}$ & $8.38 \times 10^{15}$ \\
\hline 16-17 & $2.25 \times 10^{14}$ & $3.50 \times 10^{9}$ \\
\hline 13-18 & $2.19 \times 10^{15}$ & $1.51 \times 10^{16}$ \\
\hline $18-19$ & $5.25 \times 10^{20}$ & $1.39 \times 10^{9}$ \\
\hline 20-21 & 0.02 & $8.12 \times 10^{13}$ \\
\hline $20-22$ & 4024.67 & $1.65 \times 10^{20}$ \\
\hline $22-23$ & $4.56 \times 10^{6}$ & $2.73 \times 10^{15}$ \\
\hline $23-24$ & $1.43 \times 10^{14}$ & $7.78 \times 10^{8}$ \\
\hline $20-25$ & $1.77 \times 10^{14}$ & $4.55 \times 10^{14}$ \\
\hline $25-26$ & $5.25 \times 10^{20}$ & $2.37 \times 10^{10}$ \\
\hline 20-27 & 11.37 & $8.44 \times 10^{9}$ \\
\hline $27-29$ & $8.39 \times 10^{11}$ & $8.07 \times 10^{16}$ \\
\hline $29-30$ & $5.86 \times 10^{7}$ & $8.07 \times 10^{16}$ \\
\hline $27-28$ & $5.25 \times 10^{20}$ & $2.28 \times 10^{7}$ \\
\hline 28-31 & $1.84 \times 10^{14}$ & $3.43 \times 10^{11}$ \\
\hline $30-31$ & $1.83 \times 10^{9}$ & $9.51 \times 10^{10}$ \\
\hline 31-32 & $4.88 \times 10^{14}$ & $2.77 \times 10^{12}$ \\
\hline $32-33$ & $5.25 \times 10^{20}$ & 0.01 \\
\hline $\mathrm{CF}_{3}-\mathrm{CHF}_{3}$ & $6.65 \times 10^{11}$ & $4.76 \times 10^{14}$ \\
\hline $\mathrm{CF}_{3}-\mathrm{CF}_{3} \mathrm{OH}$ & $5.25 \times 10^{20}$ & 0.005 \\
\hline $20-34$ & $6.47 \times 10^{-12}$ & $4.47 \times 10^{13}$ \\
\hline 34-35 & $5.25 \times 10^{20}$ & 0.83 \\
\hline F-HF & $3.02 \times 10^{14}$ & $4.79 \times 10^{9}$ \\
\hline $36-37$ & $1.75 \times 10^{-8}$ & $2.44 \times 10^{14}$ \\
\hline $37-(38 a+38 b)$ & $3.50 \times 10^{8}$ & $3.75 \times 10^{7}$ \\
\hline 36-39 & $6.24 \times 10^{11}$ & $5.89 \times 10^{15}$ \\
\hline $39-40$ & $4.27 \times 10^{18}$ & $1.40 \times 10^{14}$ \\
\hline $40-41$ & $5.25 \times 10^{20}$ & $3.30 \times 10^{14}$ \\
\hline $41-42$ & $3.30 \times 10^{14}$ & $4.55 \times 10^{12}$ \\
\hline $42-43$ & $3.16 \times 10^{17}$ & $3.68 \times 10^{11}$ \\
\hline $43-44$ & $5.25 \times 10^{20}$ & 0.08 \\
\hline
\end{tabular}




\begin{tabular}{|c|c|c|}
\hline $\mathbf{4 4 - 4 5}$ & $3.84 \times 10^{16}$ & 10737.90 \\
\hline $\mathbf{4 5 - 4 6}$ & $2.62 \times 10^{10}$ & $2.72 \times 10^{18}$ \\
\hline $\mathbf{4 6 - 4 7}$ & $5.25 \times 10^{20}$ & $0.68 \times 10^{-3}$ \\
\hline $\mathbf{4 0 - 4 8}$ & $1.80 \times 10^{10}$ & $1.75 \times 10^{11}$ \\
\hline $\mathbf{4 8 - 4 9}$ & $5.25 \times 10^{20}$ & $0.49 \times 10^{-3}$ \\
\hline $\mathbf{3 6 - 5 0}$ & 0.04 & $7.44 \times 10^{14}$ \\
\hline
\end{tabular}

Table S18: Free energies (in au) of 20 and transition state of step $\mathbf{2 0} \rightarrow \mathbf{2 7}(\mathbf{2 0 - 2 7})$ computed at $600 \mathrm{~K}, 700 \mathrm{~K}$ and $800 \mathrm{~K}$ using MPW1K/6-31+G(d,p) level of theory.

\begin{tabular}{|c|c|c|c|}
\hline & $\mathbf{6 0 0} \mathbf{~ K}$ & $\mathbf{7 0 0} \mathbf{~}$ & $\mathbf{8 0 0} \mathbf{~ K}$ \\
\hline $\mathbf{2 0}$ & -1737.794881 & -1737.837104 & -1737.883055 \\
\hline $\mathbf{2 0 - 2 7}$ & -1737.680173 & -1737.723704 & -1737.770940 \\
\hline
\end{tabular}

Table S19: Free energy barriers (in $\mathrm{kcal} / \mathrm{mol}$ ) for the step $\mathbf{2 0} \rightarrow \mathbf{2 7}$ computed at $600 \mathrm{~K}, 700 \mathrm{~K}$ and $800 \mathrm{~K}$ using MPW1K/6$31+G(d, p)$ level of theory.

\begin{tabular}{|c|c|c|}
\hline $\mathbf{6 0 0} \mathbf{~ K}$ & $\mathbf{7 0 0} \mathbf{K}$ & $\mathbf{8 0 0 ~ K}$ \\
\hline 72.0 & 71.2 & 70.4 \\
\hline
\end{tabular}

Table S20: Enthalpy (in au) of chemical species involved in the crucial reaction pathways idetified (Figure 7 in manuscript ) for HFPE degradation computed at $600 \mathrm{~K}$ using MPW1K/6-31+G(d,p) level of theory.

\begin{tabular}{|c|c|}
\hline & Enthalpy, $H(\mathrm{au})$ \\
\hline $\mathbf{2 0}$ & -1737.553318 \\
\hline $\mathbf{2 7}$ & -1400.008779 \\
\hline $\mathbf{2 9}$ & -1475.822360 \\
\hline $\mathbf{3 0}$ & -1550.271595 \\
\hline $\mathbf{3 1}$ & -1550.902957 \\
\hline $\mathbf{3 2}$ & -1475.158023 \\
\hline $\mathbf{3 3}$ & $-962.997787+-512.131739$ \\
\hline $\mathrm{CF}_{3}$ & -337.442452 \\
\hline $\mathrm{CHF}_{3}$ & -338.106757 \\
\hline $\mathrm{CF}_{3} \mathrm{OH}$ & -413.311193 \\
\hline $\mathrm{OH}$ & -75.696655 \\
\hline $\mathrm{O}_{2}$ & -150.252137 \\
\hline $\mathrm{H}_{2} \mathrm{O}$ & -76.373886 \\
\hline
\end{tabular}


Table S21: Enthalpy of reaction , $\Delta H=H_{\text {product }}-H_{\text {reactant }}$ (in kcal mol${ }^{-1}$ ), for various elementary steps involved in the crucial reaction pathways idetified (Figure 7 in manuscript ) for HFPE degradation computed at $600 \mathrm{~K}$ using MPW1K/6$31+\mathrm{G}(\mathrm{d}, \mathrm{p})$ level of theory.

\begin{tabular}{|c|c|}
\hline Step & $\begin{array}{c}\text { Enthalpy of reaction, } \Delta H \\
\left(\mathrm{kcal} \mathrm{mol}^{-1}\right)\end{array}$ \\
\hline $\mathbf{2 0 - 2 7}$ & 64.06 \\
\hline $\mathbf{2 7 - 2 8}$ & -73.37 \\
\hline $\mathbf{2 7 - 2 9}$ & -6.70 \\
\hline $\mathbf{2 9 - 3 0}$ & 28.78 \\
\hline $\mathbf{3 0 - 3 1}$ & 30.30 \\
\hline $\mathbf{3 1 - 3 2}$ & 17.88 \\
\hline $\mathbf{3 2 - 3 3}$ & -106.64 \\
\hline $\mathbf{2 8 - 3 1}$ & -8.09 \\
\hline $\mathrm{CF}_{3-} \mathrm{CHF}$ & 8.11 \\
\hline $\mathrm{CF}_{3-} \mathrm{CF}_{3} \mathrm{OH}$ & -107.99 \\
\hline
\end{tabular}




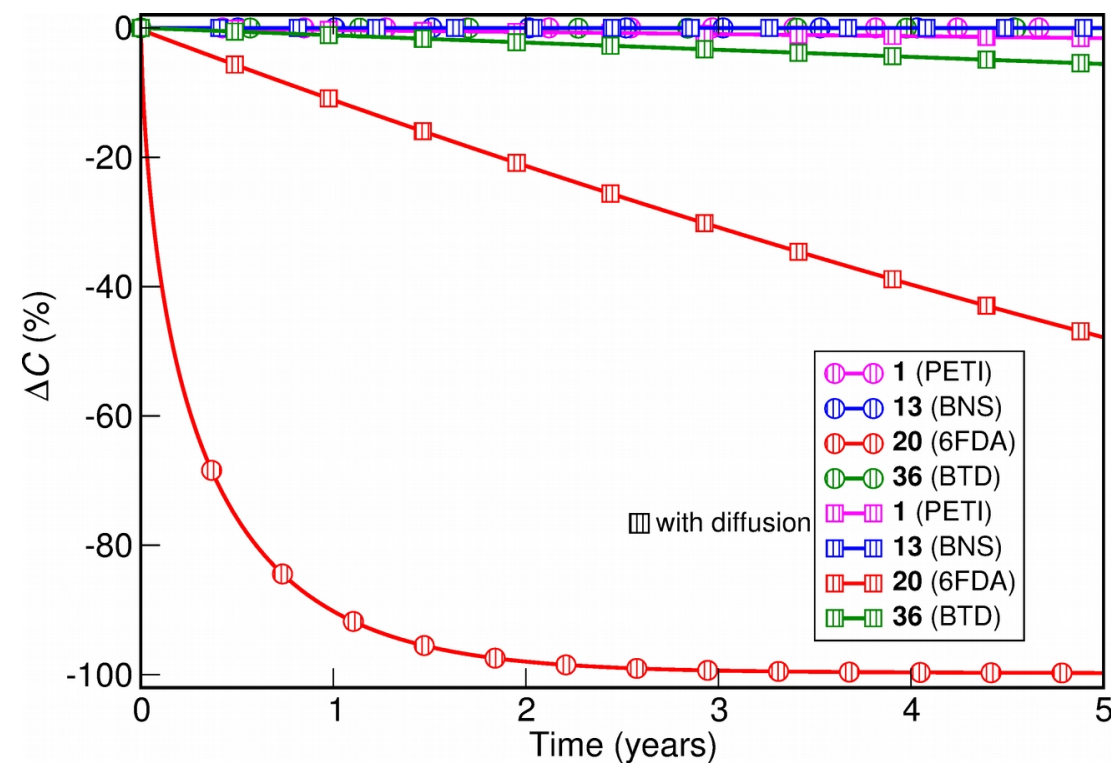

Figure S28: Percentage of decrease in concentration of different residues of HFPE during degradation, obtained from the microkinetic analysis performed with (lines with filled squares) and without (lines with filled circles) including the diffusion of radicals. Here, a rate constant of $3 \times 10^{15} \mathrm{~L} \mathrm{~mol}^{-1}$ year-1 was used to account for the diffusion of radicals in radical quenching reactions.

\section{Full Citation of Ref. 45}

Gaussian 09, Revision B.01, M. J. Frisch, G. W. Trucks, H. B. Schlegel, G. E. Scuseria, M. A. Robb, J. R. Cheeseman, G. Scalmani, V. Barone, B. Mennucci, G. A. Petersson, H. Nakatsuji, M. Caricato, X. Li, H. P. Hratchian, A. F. Izmaylov, J. Bloino, G. Zheng, J. L. Sonnenberg, M. Hada, M. Ehara, K. Toyota, R. Fukuda, J. Hasegawa, M. Ishida, T. Nakajima, Y. Honda, O. Kitao, H. Nakai, T. Vreven, J. A. Montgomery, Jr., J. E. Peralta, F. Ogliaro, M. Bearpark, J. J. Heyd, E. Brothers, K. N. Kudin, V. N. Staroverov, T. Keith, R. Kobayashi, J. Normand, K. Raghavachari, A. Rendell, J. C. Burant, S. S. Iyengar, J. Tomasi, M. Cossi, N. Rega, J. M. Millam, M. Klene, J. E. Knox, J. B. Cross, V. Bakken, C. Adamo, J. Jaramillo, R. Gomperts, R. E. Stratmann, O. Yazyev, A. J. Austin, R. Cammi, C. Pomelli, J. W. Ochterski, R. L. Martin, K. Morokuma, V. G. Zakrzewski, G. A. Voth, P. Salvador, J. J. Dannenberg, S. Dapprich, A. D. Daniels, O. Farkas, J. B. Foresman, J. V. Ortiz, J. Cioslowski, and D. J. Fox, Gaussian, Inc., Wallingford CT, 2010.

\section{Full Citation of Ref. 55}

Guimard, N. K.; Ho, J.; Brandt, J.; Lin, C. Y.; Namazian, M.; Mueller, J. O.; Oehlenschlaeger, K. K.; Hilf, S.; Lederer, A.; Schmidt, F. G.; Coote, M. L.; Barner-Kowollik, C. Harnessing Entropy to Direct the Bonding/Debonding of Polymer Systems Based on Reversible Chemistry. Chem. Sci. 2013, 4, 2752-2759. 